\title{
On Regularization of an Optimal Control Problem for Ill-Posed Nonlinear Elliptic Equations
}

\author{
Peter I. Kogut $\mathbb{D}^{1},{ }^{1}$ Olha P. Kupenko $\mathbb{D}^{2,3}$ and Rosanna Manzo $\mathbb{D}^{4}$ \\ ${ }^{1}$ Department of Differential Equations, Oles Honchar Dnipro National University, Gagarin av. 72, 49010 Dnipro, Ukraine \\ ${ }^{2}$ Department of System Analysis and Control, National Technical University "Dnipro Polytechnics", Yavornitsky av. 19, \\ 49005 Dnipro, Ukraine \\ ${ }^{3}$ Institute of Applied and System Analysis, Ihor Sikorsky National Technical University of Ukraine "Kiev Polytechnical Institute", \\ Peremogy av. 37, Build. 35, 03056 Kiev, Ukraine \\ ${ }^{4}$ Department of Information Engineering, Electrical Engineering and Applied Mathematics, University of Salerno, Via Giovanni \\ Paolo II 132, Fisciano, Italy
}

Correspondence should be addressed to Rosanna Manzo; rmanzo@unisa.it

Received 11 July 2020; Revised 24 September 2020; Accepted 26 October 2020; Published 17 November 2020

Academic Editor: Fasma Diele

Copyright (C) 2020 Peter I. Kogut et al. This is an open access article distributed under the Creative Commons Attribution License, which permits unrestricted use, distribution, and reproduction in any medium, provided the original work is properly cited.

\begin{abstract}
We discuss the existence issue to an optimal control problem for one class of nonlinear elliptic equations with an exponential type of nonlinearity. We deal with the control object when we cannot expect to have a solution of the corresponding boundary value problem in the standard functional space for all admissible controls. To overcome this difficulty, we make use of a variant of the classical Tikhonov regularization scheme. In particular, we eliminate the PDE constraints between control and state and allow such pairs run freely by introducing an additional variable which plays the role of "compensator" that appears in the original state equation. We show that this fictitious variable can be determined in a unique way. In order to provide an approximation of the original optimal control problem, we define a special family of regularized optimization problems. We show that each of these problems is consistent, well-posed, and their solutions allow to attain an optimal solution of the original problem as the parameter of regularization tends to zero. As a consequence, we prove the existence of optimal solutions to the original problem and propose a way for their approximation.
\end{abstract}

\section{Introduction}

The main object of our study is the following optimal control problem for a nonlinear elliptic equation:

$$
\text { Minimize } J(u, y)=\frac{1}{2} \int_{\Omega}\left|y-y_{d}\right|^{2} d x+\frac{\alpha}{p} \int_{\Omega}|u|^{p} d x,
$$

subject to constrains

$$
\begin{aligned}
-\Delta y & =f(y)+u \text { in } \Omega, \\
y & =0 \text { on } \partial \Omega, \\
u & \in U_{\partial} \subseteq L^{p}(\Omega), y \in H_{0}^{1}(\Omega),
\end{aligned}
$$

where $\Omega$ is a bounded open domain in $\mathbb{R}^{N}, N \geq 1$, the boundary $\partial \Omega$ is assumed to be Lipschitz, $f(y)=F^{\prime}(y)$, where $F \epsilon$ $C^{1}(\mathbb{R})$ is a given nonlinear function, $y_{d} \in L^{2}(\Omega)$ is a given distribution, $U_{\partial}$ is a nonempty closed convex subset of $L^{p}(\Omega)$, $2 \leq p<+\infty$, and $\alpha \geq 0$ is a given weight coefficient.

Optimal control governed by PDEs has been examined thoroughly since the pioneering work of J.L. Lions (see [1, 2], for instance). Other important references that also deal with the numerical approximation and in addition to those already mentioned above without any attempt to be exhaustive, are [3-9]. However, as for the optimal control problem (OCP) (1)-(4) and the corresponding Dirichlet boundary value problem (BVP) (2)-(3), it is well known that they are ill-posed, in general, and it is unknown whether the set of optimal pairs to the problem (1)-(4) is nonempty. In 
particular, there is no reason to assert the existence of weak solutions to (2)-(3) for a given $u \in L^{p}(\Omega)$ or to suppose that such solution, even if it exists, is unique (see, for instance, I.M. Gelfand [10], M.G. Crandall and P.H. Rabinowitz [11], F. Mignot and J.P. Puel [12], T. Gallouët, F. Mignot and J.P. Puel [13], H. Fujita [14], R.G. Pinsky [15], R. Ferreira, A. De Pablo, J.L. Vazquez [16], J. Dolbeault and R. Stańczy [17]).

The novelty of this paper is that we discuss the existence of optimal pairs to OCP (1)-(4) using an indirect approach based on the classical Tikhonov regularization technique in its special implementation. The idea to involve the Tikhonov regularization is inspired by the following reason: the main characteristic feature of BVP (2)-(3) is the fact that because of the specificity of nonlinearity $f(y)$ (in many particular implementations of the model (2)-(3), $F(u)=\lambda e^{u}$, [18, $19])$, we have no a priori estimate for the weak solutions in the standard Sobolev space $H_{0}^{1}(\Omega)$. As a result, the consistency of OCP (1)-(4) and existence of optimal pairs can be established only if we impose rather strict assumptions on the original data. In particular, it was shown in [20] that the set of optimal solutions of (1)-(4) is nonempty provided $N>2, p>2$, the domain $\Omega$ is star-shaped with respect to some interior point $x_{0}$, and the set of feasible pairs $\Xi$ contains at least one pair $(u, y)$ such that $f(u) \in L^{2}(\Omega)$.

Therefore, our main intention is to show that these assumptions can be essentially weakened or even eliminated. With that in mind, in the framework of Tikhonov regularization technique, we introduce the additional variable $z$ (the socalled "defect" in the state equation) into the regularized problem in order to let the pairs "control-state" $(u, y)$ run freely in the feasible space $L^{p}(\Omega) \times H_{0}^{1}(\Omega)$ so that there is no dependence of $y$ on $u$. At the same time, there is a principle difference between the standard implementation of the Tikhonov regularization of OCPs (see, for instance, [2123]) and the proposed scheme. This difference lies in the exploitation of the terms $\varepsilon\|f(y)\|_{H^{-1}(\Omega)}^{2} / 2$ and $\varepsilon\|f(y)\|_{L^{1}(\Omega)} /$ 2 in the perturbed cost functional $J_{\varepsilon}(u, y, z)$. We show that the boundedness of these terms on the set $\Xi$ of feasible solutions to the original problem plays a crucial role in the study of asymptotic behaviour of global solutions to regularized OCPs. Having introduced a special family of optimization problems, we also show that there exists an optimal solution to the original OCP that can be attained with a prescribed level of accuracy by the sequence of optimal solutions for the regularized minimization problems (for benefit of this approach and its comparison with other ones, we refer to the recent papers [24-31]).

The paper is organized as follows. In Section 2 we give some preliminaries and describe in details the characteristic features of OCP (1)-(4). The Tikhonov regularization of the original OCP is discussed in Section 3. The key result of this section is Theorem 8 , where we announce the sufficient conditions of the existence of optimal solutions to the regularized problems. In Section 4, we focus on deriving and substantiation of optimality conditions for regularized optimal control problem. The details of the indirect approach to the study of the original optimal control problem are discussed in last section. The key points of such approach are summarized in Theorem 13.

\section{Preliminaries}

Let $\Omega$ be a bounded open subset of $\mathbb{R}^{N}(N \geq 1)$. Let $F: \mathbb{R}$ $\longrightarrow[0,+\infty)$ be a mapping such that $F \in C^{1}(\mathbb{R})$. We specify this mapping as follows: there exists a constant $C_{F}>0$ such that

$$
C_{F} F^{\prime}(z) \geq F(z), \forall z \in \mathbb{R} \text {,and }\left|\int_{-\infty}^{0} z F^{\prime}(z) d z\right|<+\infty .
$$

Then, it is easy to deduce that

$$
\begin{aligned}
& F(z) \geq F(a) \exp \left(C_{F}^{-1}(z-a)\right), \\
& \forall a, z:-\infty<a \leq z<+\infty .
\end{aligned}
$$

Following the standard notation, by $H_{0}^{1}(\Omega)$, we denote the Sobolev space as the closure of $C_{0}^{\infty}(\Omega)$ with respect to the norm $\left(\int_{\Omega}|\nabla y|^{2} d x\right)^{1 / 2}$. Let $H^{-1}(\Omega)$ be the dual space to $H_{0}^{1}(\Omega)$.

In order to make a precise meaning of the weak solution to BVP (2)-(3) in the sense of distributions (or shortly, distributional solution), we begin with the following concept.

Definition 1. Let $u \in U_{\partial}$ be a given control function. We say that $y=y(u)$ is a weak solution to the boundary value problem (2)-(3) in the sense of distributions, if it belongs to the class of functions

$$
H_{f}=\left\{y \in H_{0}^{1}(\Omega): f(y) \in L_{l o c}^{1}(\Omega)\right\},
$$

and the integral identity

$$
\int_{\Omega}(\nabla y, \nabla \varphi) d x=\int_{\Omega} f(y) \varphi d x+\int_{\Omega} u \varphi d x
$$

holds for every test function $\varphi \in C_{0}^{\infty}(\Omega)$.

Since for each test function $\varphi \in C_{0}^{\infty}(\Omega)$, there exists a compact set $K \subset \Omega$ such that

$$
\operatorname{supp} \varphi \subseteq K \subset \Omega \text {, }
$$

it follows that the second term in (8) is well defined, namely,

$$
\int_{\Omega} f(y) \varphi d x \leq \int_{K}\left|f(y)\|\varphi \mid d x \leq\| \varphi\left\|_{C(\bar{K})}\right\| f(y) \|_{L^{1}(K)} .\right.
$$

At the same time, it is unknown whether the original BVP admits at least one weak solution in the sense of Definition 1 for each admissible control $u \in U_{\partial} \subseteq L^{p}(\Omega)$. Moreover, as follows from (8), the continuity of form $\varphi \mapsto[y, \varphi]_{f}:=\int_{\Omega}$ $f(y) \varphi d x$ on the set $H_{0}^{1}(\Omega)$ is not evident. For the details related with this issue, we refer to the classical paper Casas, Kavian, and Puel [20].

Before proceeding further, we make use of the following observation. Assume that for a given $u \in U_{\partial}$, we have $y \in H_{f}$, and the pair $(u, y)$ is related by integral identity (8). Then, for each test function $\varphi \in C_{0}^{\infty}(\Omega)$, the following estimate 


$$
\begin{aligned}
\int_{\Omega} f(y) \varphi d x \leq & \left|\int_{\Omega}(\nabla y, \nabla \varphi) d x\right|+\left|\int_{\Omega} u \varphi d x\right| \\
\leq & \|y\|_{H_{0}^{1}(\Omega)}\|\varphi\|_{H_{0}^{1}(\Omega)}+\|u\|_{L^{2}(\Omega)}\|\varphi\|_{L^{2}(\Omega)} \quad \text { by Poincare' }{ }^{\prime} \text { sineq. } \\
& \cdot\left[\|y\|_{H_{0}^{1}(\Omega)}+C_{\Omega}|\Omega|^{\frac{p-2}{2 p}}\|u\|_{L^{p}(\Omega)}\right]\|\varphi\|_{H_{0}^{1}(\Omega)},
\end{aligned}
$$

holds true. Hence, the mapping $\varphi \mapsto[y, \varphi]_{f}$ can be extended by continuity onto the set of all $\varphi \in H_{0}^{1}(\Omega)$ using (11) and the standard rule

$$
[y, \varphi]_{f}=\lim _{\varepsilon \rightarrow 0}\left[y, \varphi_{\varepsilon}\right]_{f}
$$

where $\left\{\varphi_{\varepsilon}\right\}_{\varepsilon>0} \subset C_{0}^{\infty}\left(\mathbb{R}^{N}\right)$ and $\varphi_{\varepsilon} \longrightarrow \varphi$ strongly in $H_{0}^{1}(\Omega)$ as $\varepsilon \longrightarrow 0$. In particular, if $y \in H_{f}$, then we can define the value $[y, y]_{f}$, and this one is finite for every $y \in H_{f}$. As a consequence, we deduce: if $y \in H_{f}$ is a weak solution to boundary value problem (2)-(3), then $y$ satisfies the energy equality

$$
\int_{\Omega}|\nabla y|^{2} d x=[y, y]_{f}+\int_{\Omega} u y d x
$$

However, it is unknown whether the value $[y, y]_{f}$ preserves a constant sign for all $y \in H_{f}$. Therefore, we cannot make use of the energy equality (13) in order to derive a priori estimate in $\|\cdot\|_{\left.H^{1} \Omega\right)}$-norm for the weak solutions.

In particular, to specify the term $[y, y]_{f}$, we have the following result (we refer to [20], Lemma 2.1) where this result was proven for a particular nonlinearity $f(y)=e^{y}$ (see also $[27,28,32]$ for the more general cases).

Lemma 2. Let $y=y(u)$ be a weak solution to $B V P(2)-(3)$ for a given $u \in U_{\partial}$. Then, $f(y) \in H^{-1}(\Omega)$,

$$
\|f(y)\|_{H^{-1}(\Omega)} \leq\|y\|_{H_{0}^{l}(\Omega)}+C_{\Omega}|\Omega|^{(p-2) / 2 p}\|u\|_{L^{p}(\Omega)},
$$

$$
[y, z]_{f}=\left\langle f(y), z>_{H^{-1}(\Omega) ; H_{0}^{1}(\Omega)}=\int_{\Omega} z f(y) d x, \forall z \in H_{0}^{1}(\Omega)\right.
$$

and, therefore, $z f(y) \in L^{1}(\Omega)$ for every $z \in H_{0}^{1}(\Omega)$.

Proof. Taking into account the Friedrich's inequality

$$
\|y\|_{L^{2}(\Omega)} \leq C_{\Omega}\|\nabla y\|_{L^{2}(\Omega)^{N}}, \forall y \in H_{0}^{1}(\Omega)
$$

and following the definition of the weak solution, we have (see (8))

$$
\begin{aligned}
& \int_{\Omega} f(y) \varphi d x \leq\left|\int_{\Omega}(\nabla y, \nabla \varphi) d x\right|+\left|\int_{\Omega} u \varphi d x\right| \\
& \leq\|\nabla y\|_{L^{2}(\Omega)}\|\nabla \varphi\|_{L^{2}(\Omega)}+\|u\|_{L^{2}(\Omega)}\|\varphi\|_{L^{2}(\Omega)} \\
& \stackrel{\operatorname{by}(13)}{\leq}\|y\|_{H_{0}^{1}(\Omega)}\|\varphi\|_{H_{0}^{1}(\Omega)}+C_{\Omega}|\Omega|^{(p-2) / 2 p} \\
& \cdot\|u\|_{L^{p}(\Omega)}\|\varphi\|_{H_{0}^{1}(\Omega)}, \forall \varphi \in C_{0}^{\infty}\left(\mathbb{R}^{N}\right)
\end{aligned}
$$

Hence, $y \in H_{f}$ by Definition 1 .

Let $z \in H_{0}^{1}(\Omega) \cap L^{\infty}(\Omega)$ be an arbitrary element. Since $f(y) \in L^{1}(\Omega)$, it follows that the term $\int_{\Omega} z f(y) d x$ is well defined. Let $\left\{\varphi_{\varepsilon}\right\}_{\varepsilon>0} \subset C^{\infty}(\bar{\Omega})$ be a sequence such that $\varphi_{\varepsilon}$ $\longrightarrow z$ in $H_{0}^{1}(\Omega)$. Moreover, in this case, we can suppose that

$$
\sup _{\varepsilon>0}\left\|\varphi_{\varepsilon}\right\|_{L^{\infty}(\Omega)}<+\infty \text { and } \varphi_{\varepsilon} \stackrel{*}{\rightarrow} z \text { in } L^{\infty}(\Omega) .
$$

Hence, due to the fact that $y \in H_{f}$, we get

$$
\int_{\Omega} z f(y) d x=\lim _{\varepsilon \rightarrow 0} \int_{\Omega} \varphi_{\varepsilon} f(y) d x=\lim _{\varepsilon \rightarrow 0}\left[y, \varphi_{\varepsilon}\right]_{f} \stackrel{\text { by }(18)}{=}[y, z]_{f} .
$$

Thus, we arrive at relation (15) for each $z \in H_{0}^{1}(\Omega) \cap L^{\infty}$ $(\Omega)$.

Let us take now $z \in H_{0}^{1}(\Omega)$ such that $z \geq 0$ almost everywhere in $\Omega$. For every $\varepsilon>0$, let $T_{\varepsilon}: \mathbb{R} \longrightarrow \mathbb{R}$ be the truncation operator defined by

$$
T_{\varepsilon}(s)=\max \left\{\min \left\{s, \varepsilon^{-1}\right\},-\mathcal{E}^{-1}\right\}
$$

The following property of $T_{\varepsilon}$ is well known (see [33]): if $z \in H_{0}^{1}(\Omega)$, then

$$
\begin{aligned}
T_{\varepsilon}(z) & \in L^{\infty}(\Omega) \cap H_{0}^{1}(\Omega) \forall \varepsilon \\
& >0 \text { and } T_{\varepsilon}(z) \longrightarrow z \text { in } H_{0}^{1}(\Omega) \text { as } \varepsilon \longrightarrow 0
\end{aligned}
$$

Hence, $T_{\varepsilon}(z) \longrightarrow z$ almost everywhere in $\Omega$. Since

$$
T_{\varepsilon}(z) f(y) \stackrel{\text { by }(5)}{\geq} \frac{1}{C_{F}} T_{\varepsilon}(z) F(y) \geq 0 \text { in } \Omega,
$$

it follows that $\left\{T_{\varepsilon}(z) f(y)\right\}_{\mathcal{E}>0}$ is a pointwise nondecreasing sequence, and also, $T_{\varepsilon}(z) f(y) \longrightarrow z f(y)$ for almost all $x \in$ $\Omega$. Therefore, by monotone convergence theorem, $z f(y)$ is a measurable function on $\Omega$, and

$$
\lim _{\varepsilon \rightarrow 0} \int_{\Omega} T_{\varepsilon}(z) f(y) d x=\int_{\Omega} z f(y) d x
$$

Thus, (15) holds true for each $z \in H_{0}^{1}(\Omega)$ such that $z \geq 0$.

As for a general case, i.e., $z \in H_{0}^{1}(\Omega)$, it is enough to note that $z=z^{+}-z^{-}$with $z^{+}, z^{-} \in H_{0}^{1}(\Omega)$ and $z^{+}, z^{-} \geq 0$ in $\Omega$, where $z^{+}:=\max \{z, 0\}, z^{-}:=\max \{-z, 0\}$. 
To complete the proof, it remains to observe that

$$
\begin{aligned}
& \int_{\Omega} z f(y) d x \stackrel{\text { by }(17)}{=} \lim _{\varepsilon \rightarrow 0} \int_{\Omega} \varphi_{\varepsilon} f(y) d x \\
& \stackrel{\text { by(17)(14) }}{\leq} \lim _{\varepsilon \rightarrow 0}\left(\|y\|_{H_{0}^{1}(\Omega)}^{p-1}+|\Omega|^{(p-2) / 2 p} C_{\Omega}\|u\|_{L^{p}(\Omega)}\right)\left\|\varphi_{\varepsilon}\right\|_{H_{0}^{1}(\Omega)} \\
& \text { - (by the strong convergence of } \varphi_{\varepsilon} \longrightarrow \mathrm{z} \text { in } H_{0}^{1}(\Omega) \text { ) } \\
& =\left(\|y\|_{H_{0}^{1}(\Omega)}^{p-1}+|\Omega|^{(p-2) / 2 p} C_{\Omega}\|u\|_{L^{p}(\Omega)}\right)\|z\|_{H_{0}^{1}(\Omega)},
\end{aligned}
$$

holds true for an arbitrary element $z \in H_{0}^{1}(\Omega)$. As a result, we have $f(y) \in H^{-1}(\Omega)$ and

$$
\begin{aligned}
\langle f(y), z\rangle_{H^{-1}(\Omega) ; H_{0}^{1}(\Omega)} & =\int_{\Omega} z f(y) d x, \forall z \in H_{0}^{1}(\Omega), \text { and }\|f(y)\|_{H^{-1}(\Omega)} \\
& \leq\left(\|y\|_{H_{0}^{1}(\Omega)}^{p-1}+|\Omega|^{(p-2) / 2 p} C_{\Omega}\|u\|_{L^{p}(\Omega)}\right) .
\end{aligned}
$$

Remark 3. As follows from Lemma 2, whenever $(y, u)$ is related by integral identity (8) and $y \in H_{f}$, then $f(y) \in$ $H^{-1}(\Omega)$, but for a general $\varphi \in H_{0}^{1}(\Omega)$, it is not necessarily true that the duality action $\langle f(y), \varphi\rangle_{H^{-1}(\Omega) ; H_{0}^{1}(\Omega)}$ is given by an integral $\int_{\Omega} \varphi f(y) d x$, hence the need for a rigorous definition of $[y, \varphi]_{f}$.

As a direct consequence of Lemma 2 and relation (13), we can specify the energy equality (13) as follows.

Corollary 4. Let $u \in U_{\partial}$ be a given control and let $y=y(u) \in$ $H_{0}^{1}(\Omega)$ be a weak solution to BVP (2)-(3) in the sense of Definition 1. Then, the energy equality for $y$ takes the form

$$
\int_{\Omega}|\nabla y|^{2} d x=\int_{\Omega} y f(y) d x+\int_{\Omega} u y d x
$$

Since it is unknown whether there exists a weak solution to BVP (2)-(3) for a given $u \in U_{\partial}$, or to suppose that such solution, even if it exists, is unique, it motivates us to introduce the following set.

Definition 5. We say that a pair $(u, y)$ is a feasible solution for optimal control problem (1)-(4) if $u \in U_{\partial}, y \in H_{f}, f(y) \in L^{1}$ $(\Omega)$, and the pair $(u, y)$ is related by integral identity (8). By $\Xi \subset L^{p}(\Omega) \times H_{0}^{1}(\Omega)$, we denote the set of all feasible solutions.

As for the optimal control problem (1)-(4), it was mentioned in [20] that its study is a nontrivial matter because of the specific of nonlinearity $f(y)$ (in [20], the authors consider the case $\left.f(y)=e^{y}\right)$. The main troubles in this case are strongly related with the following circumstances:

(i) The set of feasible solutions can be empty, in general

(ii) Even if $\Xi \neq \varnothing$, we have no a priori estimate for the weak solutions of (2)-(3) with arbitrary $u \in U_{\partial}$ (iii) Some a priori estimates can be established if only $N>2$, the domain $\Omega$ has a sufficiently smooth boundary, and it is star-shaped with respect to some interior point $x_{0}$, i.e,

$$
\left(\sigma-x_{0}, \nu(\sigma)\right) \geq 0 \text {, for a.a. } \sigma \in \partial \Omega \text {, }
$$

where $v(\sigma)$ denotes the outward unit normal vector to $\partial \Omega$ at the point $\sigma$, and the considered weak solutions $y(u)$ of (2)-(3) satisfies the extra property $f(y)$ $\in L^{2}(\Omega)$

(iv) Since we have no estimates for the states (especially without the above mentioned extra property $f(y) \epsilon$ $\left.L^{2}(\Omega)\right)$, it follows that we cannot deduce the boundedness in $L^{p}(\Omega) \times H_{0}^{1}(\Omega)$ of a minimizing sequence to the problem (1)-(4)

(v) Even if a minimizing sequence $\left\{\left(u_{k}, y_{k}\right) \in \Xi\right\}_{n \in \mathbb{N}}$ is weakly compact in $L^{p}(\Omega) \times H_{0}^{1}(\Omega)$ with $p \geq 2$, it does not allow to pass to the limit in integral identity (8) as $k \longrightarrow \infty$, and, therefore, we are not able to prove the existence of an optimal pair to the problem (1)-(4).

Although this list can be extended by many other options, we can summarize this issue by the following existence result (in order to prove this assertion, it is enough to closely follow the arguments of the proofs of Theorems 3.5 and 3.6 in [20]).

Theorem 6. Let us assume that the following conditions hold true: $N>2, p>2$, the domain $\Omega$ has a $C^{0,1}$ boundary, this domain is star-shaped with respect to some interior point $x_{0}$, and the set of feasible pairs $\Xi$ contains at least one pair $(u, y)$ such that $f(u) \in L^{2}(\Omega)$. Then, there exists a unique pair $\left(u^{0}, y^{0}\right) \in \Xi$ such that

$$
\left(u^{0}, y^{0}\right) \in \Xi_{0} \subset \Xi, J\left(u^{0}, y^{0}\right)=\inf _{(u, y) \in \Xi_{0}} J(u, y),
$$

where

$$
\Xi_{0}=\left\{\begin{array}{l|l}
(u, y) \in \Xi & \begin{array}{l}
\left(\frac{N}{2}-1\right) \int_{\Omega}|\nabla y|^{2} d x \leq N \int_{\Omega} F(y) d x \\
-\int_{\Omega} u(x)\left(x-x_{0}, \nabla y(x)\right) d x
\end{array}
\end{array}\right\}
$$

In spite of the fact that not every pair $(u, y)$ of $\Xi_{0}$ $\subseteq \Xi$ needs to be a feasible pair to (1)-(4) with the extra property $f(y) \in L^{2}(\Omega)$, and constrained minimization problems

$$
\left\langle\inf _{(u, y) \in \Xi} J(u, y)\right\rangle \text { and }\left\langle\inf _{(u, y) \in \Xi_{0}} J(u, y)\right\rangle \text {, }
$$


are distinguished from a formal point of view, we can deduce the following result (for the proof we refer to [20], Proposition 3.2).

Proposition 7. Assume that $N>2$ and $\Omega$ is star-shaped with respect to some interior point $x_{0}$. Assume also that boundary value problem (2)-(3) has a weak solution for some control $u \in L^{p}(\Omega)$ with $p \geq 2$. Then, there exists a solution $z$ of (2)-(3) corresponding to the same control $u$ and such that

$$
\left(\frac{N}{2}-1\right) \int_{\Omega}|\nabla z|^{2} d x \leq N \int_{\Omega} F(z) d x-\int_{\Omega} u(x)\left(x-x_{0}, \nabla z(x)\right) d x .
$$

In the next section, we will show that the main restrictions coming from Theorem 6 and Proposition 7 can be eliminated by introducing a new additional variable $z$ into the problem which lets pairs $(u, y)$ run freely in the feasible space $L^{p}(\Omega) \times H_{0}^{1}(\Omega)$ so that there is no dependence of $y$ on $u$.

\section{On the Tikhonov Regularization of the Original OCP}

Let us introduce the Tikhonov regularized optimal control problem associated to the original OCP (1)-(4). Let $\varepsilon>0$ be a given small parameter. Then, the regularized problem reads as follows (for comparison, we refer to $[22,23]$ ).

$$
\text { Minimize } \begin{aligned}
J_{\varepsilon}(u, y, z)= & \frac{1}{2} \int_{\Omega}\left|y-y_{d}\right|^{2} d x \\
& +\frac{\alpha}{p} \int_{\Omega}|u|^{p} d x+\frac{1}{2 \varepsilon} \int_{\Omega}|\nabla z|^{2} d x \\
& +\frac{\varepsilon}{2}\left[\|f(y)\|_{H^{-1}(\Omega)}^{2}+\|f(y)\|_{L^{1}(\Omega)}\right],
\end{aligned}
$$

subject to constraints

$$
\begin{gathered}
u \in U_{\partial} \subseteq L^{p}(\Omega), y \in H_{0}^{1}(\Omega), f(y) \in L^{1}(\Omega) \cap H^{-1}(\Omega), \\
-\Delta z=\Delta y+f(y)+u \operatorname{in} \Omega, \\
z=0 \text { on } \partial \Omega .
\end{gathered}
$$

To begin with, let us stress again that the main reason to introduce the additional variable $z$ into the regularized problem is to let pairs $(u, y)$ run freely in the feasible space $L^{p}($ $\Omega) \times H_{0}^{1}(\Omega)$ so that there is no dependence of $y$ on $u$. On the other hand, there is a principle difference between the standard scheme of the Tikhonov regularization of OCPs (see, for instance, $[22,23]$ ) and the proposed regularization in the form (32)-(35). This difference lies in the exploitation of the terms $\varepsilon\|f(y)\|_{H^{-1}(\Omega)}^{2} / 2$ and $\varepsilon\|f(y)\|_{L^{1}(\Omega)} / 2$ in the perturbed cost functional $J_{\varepsilon}(u, y, z)$. As it will be shown later on, the boundedness of these terms on the set $\Xi$ of feasible solutions to the original problem (see Lemma 2) plays a crucial role in the study of asymptotic behaviour of global solutions $\left\{\left(u_{\varepsilon}^{0}, y_{\varepsilon}^{0}, z_{\varepsilon}^{0}\right)\right\}_{0<\varepsilon \leq \varepsilon_{0}}$ as $\varepsilon$ tends to zero.
Our main assumptions are:

(a) $\Omega$ is a bounded open domain in $\mathbb{R}^{N}, N \geq 1$

(b) $U_{\partial}$ is a nonempty closed convex subset of $L^{p}(\Omega), 2$ $\leq p<+\infty$

(c) $f: \mathbb{R} \longrightarrow[0,+\infty)$ is a given monotonically increasing mapping such that $f \in C(\mathbb{R})$.

We say that a tuple $(u, y, z) \in L^{p}(\Omega) \times\left[H_{0}^{1}(\Omega)\right]^{2}$ is a feasible solution to regularized problem (32)-(35) (in symbols, $\left.(u, y, z) \in \Lambda_{\varepsilon}\right)$, if $u \in U_{\partial}, J_{\varepsilon}(u, y, z)<+\infty$, and the following variational equality

$$
a(z, \varphi)=\mathscr{F}(\varphi)
$$

holds true for all $\varphi \in H_{0}^{1}(\Omega)$, where

$$
\mathscr{F}(\varphi):=-(\nabla y, \nabla \varphi)_{L^{2}(\Omega)^{N}}+(u, \varphi)_{L^{2}(\Omega)}+\int_{\Omega} f(y) \varphi d x
$$

and $a: H_{0}^{1}(\Omega) \times H_{0}^{1}(\Omega) \longrightarrow \mathbb{R}$ denotes the bilinear form

$$
a(z, \varphi)=\int_{\Omega}(\nabla z, \nabla \varphi) d x
$$

Let us show that, for each $\varepsilon>0$, the set of feasible solutions $\Lambda_{\varepsilon}$ to regularized problem (32)-(35) is nonempty. Indeed, let $(u, y)$ be an arbitrary pair in $L^{p}(\Omega) \times$ $H_{0}^{1}(\Omega)$ such that

$$
u \in U_{\partial} \text { and } f(y) \in L^{1}(\Omega) \cap H^{-1}(\Omega) .
$$

Then, the right hand side of (36) is well defined for each test function $\varphi \in H_{0}^{1}(\Omega)$ and satisfies the following estimate (see (12)).

$$
\begin{aligned}
|\mathscr{F}(\varphi)| \leq & \left|(\nabla y, \nabla \varphi)_{L^{2}(\Omega)^{N}}\right|+\left|(u, \varphi)_{L^{2}(\Omega)}\right| \\
& +\left|\langle f(y), \varphi\rangle_{H^{-1}(\Omega) ; H_{0}^{1}(\Omega)}\right| \\
\leq & {\left[\|y\|_{H_{0}^{1}(\Omega)}+\|f(y)\|_{H^{-1}(\Omega)}\right.} \\
& \left.+C_{\Omega}|\Omega|^{(p-2) / 2 p}\|u\|_{L^{p}(\Omega)}\right]\|\varphi\|_{H_{0}^{1}(\Omega)} .
\end{aligned}
$$

Since, the bilinear form $a(\cdot, \cdot)$ is continuous and uniformly coercive on $H_{0}^{1}(\Omega) \times H_{0}^{1}(\Omega)$, it follows from LaxMilgram theorem that the variational problem (36) has a unique solution $z=z(u, y) \in H_{0}^{1}(\Omega)$. Hence, $J_{\varepsilon}(u, y, z)<+$ $\infty$ and, therefore, $(u, y, z) \in \Lambda_{\varepsilon}$ for a given $\varepsilon>0$. Thus, $\Lambda_{\varepsilon} \neq \varnothing$ and this implies that regularized optimal control problem (32)-(35) is consistent for all $\varepsilon>0$.

Our next intention is to discuss the issue related to the existence of optimal solutions of the regularized problems (32)-(35). 
Theorem 8. Assume that conditions (a)-(c) indicated before are valid. Then, for each $\varepsilon>0$, there is a triplet $\left(u_{\varepsilon}^{0}, y_{\varepsilon}^{0}, z_{\varepsilon}^{0}\right) \in$ $\Lambda_{\varepsilon}$ such that

$$
J_{\varepsilon}\left(u_{\varepsilon}^{0}, y_{\varepsilon}^{0}, z_{\varepsilon}^{0}\right)=\inf _{(u, y, z) \in \Lambda_{\varepsilon}} J_{\varepsilon}(u, y, z)
$$

Proof. Let $\varepsilon>0$ be a given value. Since the cost functional $J_{\varepsilon}$ $: \Lambda_{\varepsilon} \longrightarrow \mathbb{R}$ is nonnegative on $\Lambda_{\varepsilon}$, it follows that there exist a $\mu_{\varepsilon} \geq 0$ and a sequence $\left\{\left(u_{\varepsilon, k}, y_{\varepsilon, k}, z_{\varepsilon, k}\right)\right\}_{k \in \mathbb{N}} \subset \Lambda_{\varepsilon}$ such that

$$
\begin{aligned}
& \mu_{\varepsilon}=\inf _{(u, y, z) \in \Lambda_{\varepsilon}} J_{\varepsilon}(u, y, z)=\lim _{k \rightarrow \infty} J_{\varepsilon}\left(u_{\varepsilon, k}, y_{\varepsilon, k}, z_{\varepsilon, k}\right) \\
& \mu_{\varepsilon} \leq J_{\varepsilon}\left(u_{\varepsilon, k+1}, y_{\varepsilon, k+1}, z_{\varepsilon, k+1}\right) \leq J_{\varepsilon}\left(u_{\varepsilon, k}, y_{\varepsilon, k}, z_{\varepsilon, k}\right) \leq \mu_{\varepsilon}+1, \forall k \in \mathbb{N} .
\end{aligned}
$$

Then, we can immediately deduce from (43) and definition of the set $\Lambda_{\varepsilon}$ that $y_{\varepsilon, k} \in H_{0}^{1}(\Omega)$ for each $k \in \mathbb{N}$ and the sequences

$$
\left\{y_{\varepsilon, k}\right\}_{k \in \mathbb{N}},\left\{u_{\varepsilon, k}\right\}_{k \in \mathbb{N}},\left\{z_{\varepsilon, k}\right\}_{k \in \mathbb{N}} \text {, and }\left\{f\left(y_{\varepsilon, k}\right)\right\}_{k \in \mathbb{N}},
$$

are uniformly bounded in $L^{2}(\Omega), L^{p}(\Omega), H_{0}^{1}(\Omega)$, and $H^{-1}(\Omega) \cap L^{1}(\Omega)$, respectively. In particular,

$$
\sup _{k \in \mathbb{N}}\left\|u_{\varepsilon, k}\right\|_{L^{p}(\Omega)}^{p} \leq \frac{\left(\mu_{\varepsilon}+1\right) p}{\alpha}, \sup _{k \in \mathbb{N}}\left\|z_{\varepsilon, k}\right\|_{H_{0}^{1}(\Omega)}^{2} \leq 2 \varepsilon\left(\mu_{\varepsilon}+1\right),
$$

$\sup _{k \in \mathbb{N}}\left\|y_{\varepsilon, k}\right\|_{L^{2}(\Omega)}^{2} \leq 4\left(\mu_{\varepsilon}+1\right)+2\left\|y_{d}\right\|_{L^{2}(\Omega)}^{2} \sup _{k \in \mathbb{N}}\left\|f\left(y_{\varepsilon, k}\right)\right\|_{H^{-1}(\Omega)}^{2} \leq \frac{2\left(\mu_{\varepsilon}+1\right)}{\varepsilon}$,

$$
\sup _{k \in \mathbb{N}}\left\|f\left(y_{\varepsilon, k}\right)\right\|_{L^{1}(\Omega)} \leq \frac{2\left(\mu_{\varepsilon}+1\right)}{\varepsilon} .
$$

Hence, without loss of generality, we can suppose that there exist elements $y_{\varepsilon} \in L^{2}(\Omega), u_{\varepsilon} \in L^{p}(\Omega), \quad \xi_{\varepsilon} \in H^{-1}(\Omega)$, and $z_{\varepsilon} \in H_{0}^{1}(\Omega)$ such that

$$
\begin{aligned}
y_{\varepsilon, k} & \rightarrow y_{\varepsilon} \text { in } L^{2}(\Omega), \\
u_{\varepsilon, k} & \rightarrow u_{\varepsilon} \text { in } L^{p}(\Omega), \\
z_{\varepsilon, k} & \rightarrow z_{\varepsilon} \text { in } H_{0}^{1}(\Omega), \\
f\left(y_{\varepsilon, k}\right) & \rightarrow \xi_{\varepsilon} \text { in } H^{-1}(\Omega),
\end{aligned}
$$

as $k \longrightarrow \infty$.

Let us show that, in fact, $y_{\varepsilon, k} \rightarrow y_{\varepsilon}$ in $H_{0}^{1}(\Omega)$. Indeed, from (36), using $y_{\varepsilon, k} \in H_{0}^{1}(\Omega)$ as a test function, we find that

$$
\begin{aligned}
& \int_{\Omega}\left[\left|\nabla y_{\varepsilon, k}\right|^{2}+\left(\nabla z_{\varepsilon, k}, \nabla y_{\varepsilon, k}\right)-u_{\varepsilon, k} y_{\varepsilon, k}\right] d x \\
& \quad=\left\langle f\left(y_{\varepsilon, k}\right), y_{\varepsilon, k}\right\rangle_{H^{-1}(\Omega) ; H_{0}^{1}(\Omega)}
\end{aligned}
$$

for all $k \in \mathbb{N}$. Then, utilizing the Poincaré's inequality, we obtain

$$
\begin{aligned}
\left\|\nabla y_{\varepsilon, k}\right\|_{L^{2}(\Omega)^{N}}^{2} \leq & {\left[\left\|z_{\varepsilon, k}\right\|_{H_{0}^{1}(\Omega)}+\left\|f\left(y_{\varepsilon, k}\right)\right\|_{H^{-1}(\Omega)}\right.} \\
& \left.+C_{\Omega}|\Omega|^{(p-2) / 2 p}\left\|u_{\varepsilon, k}\right\|_{L^{p}(\Omega)}\right] \times\left\|\nabla y_{\varepsilon, k}\right\|_{L^{2}(\Omega)^{N}} .
\end{aligned}
$$

From this and estimates (45)-(46), we deduce that

$$
\sup _{k \in \mathbb{N}}\left\|\nabla y_{\varepsilon, k}\right\|_{L^{2}(\Omega)^{N}} \leq \sqrt{\frac{2\left(\mu_{\varepsilon}+1\right)}{\varepsilon}}\left[1+\varepsilon+C_{\Omega}|\Omega|^{(p-2) / 2 p} \sqrt{\frac{p \mathcal{\varepsilon}}{2 \alpha}}\right]=: C_{\varepsilon}^{*} .
$$

Thus, without loss of generality, we can suppose that (up to a subsequence)

$y_{\varepsilon, k} \rightarrow y_{\varepsilon}$ in $H_{0}^{1}(\Omega), y_{\varepsilon, k} \longrightarrow y_{\varepsilon}$ in $L^{2}(\Omega), y_{\varepsilon, k}(x) \longrightarrow y_{\varepsilon}(x)$ a.e.in $\Omega$.

Utilizing the pointwise convergence $(55)_{3}$ and assumption $(c)$, we see that

$$
f\left(y_{\varepsilon, k}\right) \longrightarrow f\left(y_{\varepsilon}\right) \text { almost everywhere in } \Omega \text { ask } \longrightarrow \infty \text {. }
$$

Let us show that this fact together with (47) implies the strong convergence

$$
f\left(y_{\varepsilon, k}\right) \longrightarrow f\left(y_{\varepsilon}\right) \text { in } L^{1}(\Omega) \text { as } k \longrightarrow \infty .
$$

To begin with, let us show that the sequence $\left\{f\left(y_{\varepsilon, k}\right) y_{\varepsilon, k}\right\}_{k \in \mathbb{N}}$ is bounded in $L^{1}(\Omega)$. With that in mind, for each $k \in \mathbb{N}$, we make use of the decomposition $y_{\varepsilon, k}=y_{\varepsilon, k}^{+}$ $-y_{\varepsilon, k}^{-}$with

$y_{\varepsilon, k}^{+}=\max \left\{0, y_{\varepsilon, k}\right\} \in H_{0}^{1}(\Omega), y_{\varepsilon, k}^{-}=\max \left\{0,-y_{\varepsilon, k}\right\} \in H_{0}^{1}(\Omega)$,

and set

$$
T_{\eta}(y)(x)= \begin{cases}\eta, & \text { if } y(x)>\eta, \\ y(x), & \text { if } y(x) \leq \eta\end{cases}
$$

Then, $T_{\eta}(y) \in L^{\infty}(\Omega) \cap H_{0}^{1}(\Omega)$ for all $y \in H_{0}^{1}(\Omega)$ such that $y \geq 0$. Using $y_{\varepsilon, k}^{+} \in H_{0}^{1}(\Omega)$ as a test function in (36), we find that

$$
\begin{aligned}
\int_{\Omega} f\left(y_{\varepsilon, k}\right) T_{\eta}\left(y_{\varepsilon, k}^{+}\right) d x= & \int_{\Omega}\left(\nabla z_{\varepsilon, k}, \nabla T_{\eta}\left(y_{\varepsilon, k}^{+}\right)\right) d x \\
& +\int_{\Omega}\left(\nabla y_{\varepsilon, k}, \nabla T_{\eta}\left(y_{\varepsilon, k}^{+}\right)\right) d x \\
& -\int_{\Omega} u_{\varepsilon, k} T_{\eta}\left(y_{\varepsilon, k}^{+}\right) d x, \forall \eta \in \mathbb{N} .
\end{aligned}
$$


Since $T_{\eta}\left(y_{\varepsilon, k}^{+}\right) \longrightarrow y_{\varepsilon, k}^{+}$in $H_{0}^{1}(\Omega)$ as $\eta \longrightarrow \infty$, the limit passage in (60) as $\eta \longrightarrow \infty$ leads to the relation

$$
\begin{aligned}
\int_{\Omega} f\left(y_{\varepsilon, k}\right) y_{\varepsilon, k}^{+} d x= & \int_{\Omega}\left(\nabla z_{\varepsilon, k}, \nabla y_{\varepsilon, k}^{+}\right) d x+\int_{\Omega}\left(\nabla y_{\varepsilon, k}, \nabla y_{\varepsilon, k}^{+}\right) d x \\
& -\int_{\Omega} u_{\varepsilon, k} y_{\varepsilon, k}^{+} d x .
\end{aligned}
$$

From this and the fact that $\left\|y_{\varepsilon, k}^{+}\right\|_{H_{0}^{1}(\Omega)} \leq\left\|y_{\varepsilon, k}\right\|_{H_{0}^{1}(\Omega)}$, we deduce the estimate

$$
\begin{aligned}
\int_{\Omega} f\left(y_{\varepsilon, k}\right) y_{\varepsilon, k}^{+} d x \leq & {\left[\left\|z_{\varepsilon, k}\right\|_{H_{0}^{1}(\Omega)}+\left\|y_{\varepsilon, k}\right\|_{H_{0}^{1}(\Omega)}\right.} \\
& \left.+C_{\Omega}|\Omega|^{(p-2) / 2 p}\left\|u_{\varepsilon, k}\right\|_{L^{p}(\Omega)}\right]\left\|y_{\varepsilon, k}\right\|_{H_{0}^{1}(\Omega)} \underset{\text { by }(29),(29)(37)}{\leq} \\
& \cdot C_{\varepsilon}^{*}\left[\sqrt{2 \varepsilon\left(\mu_{\varepsilon}+1\right)}+C_{\varepsilon}^{*}+C_{\Omega}|\Omega|^{(p-2) / 2 p}\left(\frac{\left(\mu_{\varepsilon}+1\right) p}{\alpha}\right)^{1 / p}\right] .
\end{aligned}
$$

Arguing in a similar manner, it can be shown that

$$
\begin{aligned}
& \int_{\Omega} f\left(y_{\varepsilon, k}\right) y_{\varepsilon, k}^{-} d x \stackrel{\text { by(29),(29)(37) }}{\leq} \\
& \quad \cdot C_{\varepsilon}^{*}\left[\sqrt{2 \varepsilon\left(\mu_{\varepsilon}+1\right)}+C_{\varepsilon}^{*}+C_{\Omega}|\Omega|^{(p-2) / 2 p}\left(\frac{\left(\mu_{\varepsilon}+1\right) p}{\alpha}\right)^{1 / p}\right] .
\end{aligned}
$$

Since $y_{\varepsilon, k}^{+}+y_{\varepsilon, k}^{-}=\left|y_{\varepsilon, k}\right|$, it follows from (62)-(63) that there exist positive constants $C_{i, \varepsilon}, i=1,2$, independent of $k$ and $y_{\varepsilon, k}$ such that

$$
\sup _{k \in \mathbb{N}}\left\|f\left(y_{\varepsilon, k}\right) y_{\varepsilon, k}\right\|_{L^{1}(\Omega)} \leq C_{1, \varepsilon}\left(\mu_{\varepsilon}+1\right)+C_{2, \varepsilon}\left(\mu_{\varepsilon}+1\right)^{2 / p} .
$$

In order to prove the strong convergence (57), we make use of Vitali's theorem. To do so, we fix an arbitrary $\delta>0$ and take $m>0$ and $\tau>0$ such that $m>2 C_{\varepsilon}^{* * *} / \delta, \tau=\delta /$ $(2 f(m))$, where

$$
C_{\varepsilon}^{* *}:=C_{1, \varepsilon}\left(\mu_{\varepsilon}+1\right)+C_{2, \varepsilon}\left(\mu_{\varepsilon}+1\right)^{2 / p} .
$$

Then, for every measurable set $S \subset \Omega$ with Lebesgue measure $|S|<\delta$, we have

$$
\begin{aligned}
\int_{S} f\left(y_{\varepsilon, k}\right) d x= & \int_{\left\{x \in S: y_{\varepsilon, k}(x)>m\right\}} f\left(y_{\varepsilon, k}\right) d x+\int_{\left\{x \in S: y_{\varepsilon, k}(x) \leq m\right\}} f\left(y_{\varepsilon, k}\right) d x \\
\leq & \frac{1}{m} \int_{\left\{x \in S: y_{\varepsilon, k}(x)>m\right\}} y_{\varepsilon, k} f\left(y_{\varepsilon, k}\right) d x+\int_{\left\{x \in S: y_{\varepsilon, k}(x) \leq m\right\}} f(m) d x \\
\leq & \frac{1}{m} \int_{\Omega}\left|y_{\varepsilon, k}\right| f\left(y_{\varepsilon, k}\right) d x+f(m)|S| \stackrel{\text { by }(45)}{\leq} \frac{C_{\varepsilon}^{* *}}{m} \\
& +f(m)|S| \leq \frac{\delta}{2}+\frac{\delta}{2} .
\end{aligned}
$$

As a result, we see that the sequence $\left\{f\left(y_{\varepsilon, k}\right)\right\}_{k \in \mathbb{N}}$ is equiintegrable and, hence, the desired convergence (57) is a direct consequence of the pointwise convergence (56) and Vitali's convergence theorem. From this and (51), we obtain

$$
\xi_{\varepsilon}=f\left(y_{\varepsilon}\right), f\left(y_{\varepsilon}\right) \in H^{-1}(\Omega) \cap L^{1}(\Omega) .
$$

Since the set of admissible controls $U_{\partial}$ is convex and closed in $L^{p}(\Omega)$, it follows from Mazur's theorem that it is sequentially closed with respect to the weak topology of $L^{p}$ $(\Omega)$. Hence, $u_{\varepsilon} \in U_{\partial}$. Thus, in order to decide that $\left(u_{\varepsilon}, y_{\varepsilon}\right.$, $\left.z_{\varepsilon}\right)$ is a feasible solution to the regularized problems (32)-(35), it remains to show that this tuple is related by the variational equality (36). To do so, we utilize the following integral identity

$$
\begin{aligned}
& \int_{\Omega}\left[\left(\nabla y_{\varepsilon, k}, \nabla \varphi\right)+\left(\nabla z_{\varepsilon, k}, \nabla \varphi\right)-u_{\varepsilon, k} \varphi\right] d x \\
& =\int_{\Omega} f\left(y_{\varepsilon, k}\right) \varphi d x=\left\langle f\left(y_{\varepsilon, k}\right), \varphi\right\rangle_{H^{-1}(\Omega) ; H_{0}^{1}(\Omega)},
\end{aligned}
$$

which holds true for each $\varphi \in H_{0}^{1}(\Omega), k \in \mathbb{N}$, and $\varepsilon>0$. Taking into account properties (48)-(51), (67), the limit passage in (68) as $k \longrightarrow \infty$ becomes trivial. As a result, we arrive at the following relation

$$
\begin{aligned}
& \int_{\Omega}\left[\left(\nabla y_{\varepsilon}, \nabla \varphi\right)+\left(\nabla z_{\varepsilon}, \nabla \varphi\right)-u_{\varepsilon} \varphi\right] d x \\
& =\left\langle f\left(y_{\varepsilon}\right), \varphi\right\rangle_{H^{-1}(\Omega) ; H_{0}^{1}(\Omega)} \stackrel{\text { by }(46)}{=} \int_{\Omega} f\left(y_{\varepsilon}\right) \varphi d x, \forall \varphi \in H_{0}^{1}(\Omega) .
\end{aligned}
$$

Thus, $\left(u_{\varepsilon}, y_{\varepsilon}, z_{\varepsilon}\right)$ is a feasible solution to the regularized problems (32)-(35).

To conclude the proof, let us show that, in fact, the triplet $\left(u_{\varepsilon}, y_{\varepsilon}, z_{\varepsilon}\right)$ is optimal to the problem (32)-(35). Indeed, in view of the strong convergence (57) and lower semicontinuity of norms in reflexive Banach spaces $H_{0}^{1}(\Omega), H^{-1}(\Omega)$, and $L^{p}(\Omega)$ with respect to the weak convergence, passing to the limit in (42), we obtain

$$
\begin{aligned}
\mu_{\varepsilon} & =\inf _{(u, y, z) \in \Lambda_{\varepsilon}} J_{\varepsilon}(u, y, z)=\lim _{k \rightarrow \infty} J_{\varepsilon}\left(u_{\varepsilon, k}, y_{\varepsilon, k}, z_{\varepsilon, k}\right) \\
& \geq \liminf _{k \rightarrow \infty} J_{\varepsilon}\left(u_{\varepsilon, k}, y_{\varepsilon, k}, z_{\varepsilon, k}\right) \geq J_{\varepsilon}\left(u_{\varepsilon}, y_{\varepsilon}, z_{\varepsilon}\right) \\
& \geq \inf _{(u, y, z) \in \Lambda_{\varepsilon}} J_{\varepsilon}(u, y, z)=\mu_{\varepsilon} .
\end{aligned}
$$

Thus, the equality (41) holds true with $\left(u_{\varepsilon}^{0}, y_{\varepsilon}^{0}, z_{\varepsilon}^{0}\right)=$ $\left(u_{\varepsilon}, y_{\varepsilon}, z_{\varepsilon}\right)$ and, therefore, the tuple $\left(u_{\varepsilon}, y_{\varepsilon}, z_{\varepsilon}\right)$ is optimal for regularized problems (21)-(24).

\section{Optimality Conditions for Regularized Problem}

In this section, we focus on deriving of optimality conditions for regularized optimal control problem (32)-(35) corresponding to the case $U_{\partial}=L^{p}(\Omega)$. 
We begin with the following observations.

Remark 9. In spite of the natural expectations, the mapping $(u, y) \mapsto z$, where $z=z(u, y)$ is the solution of (34)-(35) associated to $u$ and $y$, is not of class $C^{1}$ from $L^{p}(\Omega) \times H_{0}^{1}(\Omega)$ $\longrightarrow H_{0}^{1}(\Omega)$. Indeed, if we assume this property, then the mapping $y \mapsto f(y)$ should be of class $C^{1}$ from $H_{0}^{1}(\Omega)$ into $H^{-1}(\Omega)$ on the subset $Y \subset H_{0}^{1}(\Omega)$ defined by

$$
Y=\left\{y \in H_{0}^{1}(\Omega): f(y) \in L^{1}(\Omega) \cap H^{-1}(\Omega)\right\} .
$$

Apart from the fact that, under the assumptions on the function $f$, it is not clear whether the set $Y$ has a nonempty interior int $(Y)$. Even if it is so, then the assumption that the mapping $y \mapsto f(y)$ is of class $C^{1}$ at some point $y_{0} \in$ int $(Y)$ would imply $f^{\prime}\left(y_{0}\right) h \in H^{-1}(\Omega)$ for all $h \in H_{0}^{1}(\Omega)$. However, when $N \geq 3$, even for $C^{\infty}$ function such as $f(y)=$ $\exp (y)$, this result does not hold.

Indeed, let us consider the case $N=3, f(y)=\exp (y)$, and $\Omega$ is the unit ball $B_{1}(0) \subset \mathbb{R}^{3}$. Then, the function $y(x)=-\lambda$ $\log (|x|)$, for $0<\lambda<5 / 2$, satisfies $y \in Y$. Now, if we set $h(x)$ $=|x|^{-\alpha} \varphi(x)$ for $0<\alpha<1 / 2$ and a function $\varphi \in C_{0}^{\infty}(\Omega)$ such that $0 \leq \varphi \leq 1$ and $\varphi(x)=1$ for $|x|<1 / 2$, we have $h \in H_{0}^{1}(\Omega)$ and $f^{\prime}(y(x)) h(x)=|x|^{-(\lambda+\alpha)} \varphi(x)$. However, $f^{\prime}(y) h \in H^{-1}(\Omega)$ when $\alpha+\lambda>5 / 2$.

Remark 10. In practice, the numerical simulation of $\|\cdot\|_{H^{-1}(\Omega)}$ -term in the cost functional is quite specific and a delicate matter. Usually, it is associated with providing a very precise numerical analysis. In order to avoid these difficulties, it makes sense to substitute the $\|\cdot\|_{H^{-1}(\Omega)}$ term in the cost functional by some equivalent norm. For instance, let $u^{*}$ be an element of $H^{-1}(\Omega)$ such that

$$
\begin{aligned}
\left\langle u^{*}, u\right\rangle_{H^{-1}(\Omega) ; H_{0}^{1}(\Omega)} & =\int_{\Omega}(F, \nabla u)_{\mathbb{R}^{N}} d x \\
& =\int_{\Omega}\left[F_{1} \frac{\partial u}{\partial x_{1}}+\cdots+F_{N} \frac{\partial u}{\partial x_{N}}\right] d x, \forall u \in H_{0}^{1}(\Omega),
\end{aligned}
$$

where $F=\left[F_{1}, \cdots, F_{N}\right]$ in $L^{2}\left(\Omega ; \mathbb{R}^{N}\right)$ is a given a vectorfunction.

It is clear that

$$
\left\|u^{*}\right\|_{H^{-1}(\Omega)} \leq \sqrt{\int_{\Omega}\left(F_{1}^{2}(x)+\cdots+F_{N}^{2}(x)\right) d x} .
$$

On the other hand, due to the Lax-Milgram theorem, the Dirichlet boundary value problem

$$
-\Delta y=u^{*} \text { in } \Omega, y=0 \text { on } \partial \Omega,
$$

has a unique solution $y=(-\Delta)^{-1} u^{*} \in H_{0}^{1}(\Omega)$ for each $u^{*} \epsilon$ $H^{-1}(\Omega)$. Moreover, in view of the energy equality

$$
\int_{\Omega}(\nabla y, \nabla y)_{\mathbb{R}^{N}} d x=\|\nabla y\|_{L^{2}\left(\Omega ; \mathbb{R}^{N}\right)}^{2}=\|y\|_{H_{0}^{1}(\Omega)}^{2}=\left\langle u^{*}, y\right\rangle_{H^{-1}(\Omega) ; H_{0}^{1}(\Omega)},
$$

which holds true for the weak solution of Dirichlet problems (74), we can deduce the following a priori estimate

$$
\|y\|_{H_{0}^{1}(\Omega)} \equiv\left\|(-\Delta)^{-1} u^{*}\right\|_{H_{0}^{1}(\Omega)} \equiv\left\|\nabla(-\Delta)^{-1} u^{*}\right\|_{L^{2}\left(\Omega ; \mathbb{R}^{N}\right)} \leq\left\|u^{*}\right\|_{H^{-1}(\Omega)} .
$$

Combining this result with (73), we obtain the following chain of inequalities for the dual norm $\|\cdot\|_{H^{-1}(\Omega)}$ in $H^{-1}(\Omega)$ :

$$
\begin{aligned}
& \left\|\nabla(-\Delta)^{-1} u^{*}\right\|_{L^{2}\left(\Omega ; \mathbb{R}^{N}\right)} \\
& \quad \leq\left\|u^{*}\right\|_{H^{-1}(\Omega)} \leq \sqrt{\int_{\Omega}\left(F_{1}^{2}(x)+\cdots+F_{N}^{2}(x)\right) d x} \\
& \quad \stackrel{\operatorname{by}(50)}{=} \sqrt{\int_{\Omega}|\nabla y|_{\mathbb{R}^{N}}^{2} d x}=\|\nabla y\|_{L^{2}\left(\Omega ; \mathbb{R}^{N}\right)} \\
& \quad=\left\|\nabla(-\Delta)^{-1} u^{*}\right\|_{L^{2}\left(\Omega ; \mathbb{R}^{N}\right)} .
\end{aligned}
$$

Hence, in this case, the standard norm in $H^{-1}(\Omega)$ is equivalent to the following one

$$
\left\|u^{*}\right\|_{H^{-1}(\Omega)}=\left\|\nabla(-\Delta)^{-1} u^{*}\right\|_{L^{2}\left(\Omega ; \mathbb{R}^{N}\right)}, \forall u^{*} \in H^{-1}(\Omega) .
$$

Taking this fact into account, in this section, we specify the cost functional (32) as follows

$$
\begin{aligned}
J_{\varepsilon}(u, y, z)= & \frac{1}{2} \int_{\Omega}\left|y-y_{d}\right|^{2} d x+\frac{\alpha}{p} \int_{\Omega}|u|^{p} d x+\frac{1}{2 \varepsilon} \int_{\Omega}|\nabla z|^{2} d x \\
& +\frac{\varepsilon}{2}\left[\left\|\nabla(-\Delta)^{-1} f(y)\right\|_{L^{2}\left(\Omega ; \mathbb{R}^{N}\right)}^{2}+\|f(y)\|_{L^{1}(\Omega)}\right] .
\end{aligned}
$$

As a result, to derive optimality conditions for regularized optimal control problem (79), (33)-(35), we apply the following reasoning. Let $\varepsilon>0$ be a fixed value. In addition to (5), we assume that $f(0) \neq 0$ and $f(y)=F^{\prime}(y)$ are a convex function for which there exists a constant $C_{M}>0$ such that

$$
\left|f\left(x_{1}+x_{2}\right)-f\left(x_{1}\right)\right| \leq C_{M} f\left(x_{1}\right)\left|\frac{f\left(x_{2}\right)}{f(0)}-1\right|, \forall x_{1}, x_{2} \in \mathbb{R} .
$$

Note that this property does not come into conflict with relation (6), and as a particular case of $f(x)$ satisfying (80) is $f(x)=C \exp (k x)$.

Let $\mathscr{K}$ be the following subset of $H_{0}^{1}(\Omega)$

$$
\mathscr{K}=\left\{y \in H_{0}^{1}(\Omega): \Delta y \in L^{p}(\Omega), f(y) \in L^{p}(\Omega)\right\} .
$$

In view of the properties of function $f$, it is unknown 
whether this set has a nonempty interior. However, taking into account that $p \geq 2$ and $2>2 N /(N+2)$, it follows from Sobolev embedding theorem that $f(y) \in H^{-1}(\Omega)$ for all $y \in$ $\mathscr{K}$.

We know that boundary value problem (34)-(35) has a unique solution $z \in H_{0}^{1}(\Omega)$ for every $u \in L^{p}(\Omega)$ and $y \in \mathscr{K}$. Let $\left(z_{\varepsilon}^{0}, u_{\varepsilon}^{0}, y_{\varepsilon}^{0}\right) \in H_{0}^{1}(\Omega) \times U_{\partial} \times \mathscr{K}$ be an optimal solution to the problem (79), (33)-(35) with $f\left(y_{\varepsilon}^{0}\right) \in L^{p}(\Omega)$ and $\Delta y_{\varepsilon}^{0} \epsilon$ $L^{p}(\Omega)$

Let $w \in C_{0}^{\infty}(\Omega)$ and $g \in C_{0}^{\infty}(\Omega)$ be arbitrary chosen functions. Then, property (80) immediately implies that

$$
f\left(y_{\varepsilon}^{0}+\lambda w\right) \in L^{p}(\Omega) \cap H^{-1}(\Omega) \text { for }|\lambda| \text { small enough } .
$$

By convexity of $f$, we have

$$
\begin{aligned}
f\left(y_{\varepsilon}^{0}+\lambda w\right) & \geq f\left(y_{\varepsilon}^{0}\right)+\lambda f^{\prime}\left(y_{\varepsilon}^{0}\right) w, f\left(y_{\varepsilon}^{0}-\lambda w\right) \\
& \geq f\left(y_{\varepsilon}^{0}\right)-\lambda f^{\prime}\left(y_{\varepsilon}^{0}\right) w .
\end{aligned}
$$

Then,

$$
f\left(y_{\varepsilon}^{0}\right)-f\left(y_{\varepsilon}^{0}-\lambda w\right) \leq \lambda f^{\prime}\left(y_{\varepsilon}^{0}\right) w \leq f\left(y_{\varepsilon}^{0}+\lambda w\right)-f\left(y_{\varepsilon}^{0}\right),
$$

where $f\left(y_{\varepsilon}^{0}\right)-f\left(y_{\varepsilon}^{0}-\lambda w\right)$ and $f\left(y_{\varepsilon}^{0}+\lambda w\right)-f\left(y_{\varepsilon}^{0}\right)$ belong to $L^{p}(\Omega) \cap H^{-1}(\Omega)$ (see (82)). From this, we deduce that

$$
f^{\prime}\left(y_{\varepsilon}^{0}\right) w \in L^{p}(\Omega) \cap H^{-1}(\Omega) .
$$

For every $\lambda \in \mathbb{R}, \lambda \neq 0$, we set

$$
\begin{aligned}
u_{\lambda}= & u_{\varepsilon}^{0}-\lambda \Delta g-\lambda \Delta w-f\left(y_{\varepsilon}^{0}+\lambda w\right) \\
& +f\left(y_{\varepsilon}^{0}\right), y_{\lambda}=y_{\varepsilon}^{0}+\lambda w, z_{\lambda}=z_{\varepsilon}^{0}+\lambda g .
\end{aligned}
$$

Then, property (80) implies that $y_{\lambda} \in \mathscr{K}$ and $-\Delta z_{\lambda}=\Delta$ $y_{\lambda}+f\left(y_{\lambda}\right)+u_{\lambda}$ in $\Omega$. So, $\left(u_{\lambda}, y_{\lambda}, z_{\lambda}\right)$ is a feasible point for the problem (32)-(35). As follows from (84)-(85), there exists an element

$$
r(w, \lambda) \in L^{p}(\Omega) \cap H^{-1}(\Omega),
$$

such that

$$
f\left(y_{\varepsilon}^{0}+\lambda w\right)=f\left(y_{\varepsilon}^{0}\right)+\lambda f^{\prime}\left(y_{\varepsilon}^{0}\right) w+r(w, \lambda),
$$

and $\|r(w, \lambda)\|_{L^{p}(\Omega) \cap H^{-1}(\Omega)}=o(|\lambda|)$ as $\lambda \longrightarrow 0$. Let us show that, for a given $w \in C_{0}^{\infty}(\Omega)$, the following extra properties hold true

$$
\frac{\left|f^{\prime}\left(y_{\varepsilon}^{0}\right) w\right|^{2}}{f\left(y_{\varepsilon}^{0}\right)} \in L^{1}(\Omega),\left\|\frac{r^{2}(w, \lambda)}{f\left(y_{\varepsilon}^{0}\right)}\right\|_{L^{p}(\Omega)}=o(\lambda) \text { as } \lambda \longrightarrow 0 .
$$

Indeed, due to property (80), we deduce from (84) and (88) that

$$
\begin{gathered}
C_{M} f\left(y_{\varepsilon}^{0}\right)\left|\frac{f(\lambda w)}{f(0)}-1\right| \geq\left|\lambda f^{\prime}\left(y_{\varepsilon}^{0}\right) w\right|, \\
C_{M} f\left(y_{\varepsilon}^{0}\right)\left|\frac{f(\lambda w)}{f(0)}-1\right| \geq|r(w, \lambda)| .
\end{gathered}
$$

Since $f(\lambda w) \in L^{\infty}(\Omega)$, the above inequalities imply that

$$
\frac{f^{\prime}\left(y_{\varepsilon}^{0}\right) w}{f\left(y_{\varepsilon}^{0}\right)} \in L^{\infty}(\Omega), \frac{r(w, \lambda)}{f\left(y_{\varepsilon}^{0}\right)} \in L^{\infty}(\Omega) .
$$

Hence,

$$
\begin{aligned}
& \left\|\frac{\left|f^{\prime}\left(y_{\varepsilon}^{0}\right) w\right|^{2}}{f\left(y_{\varepsilon}^{0}\right)}\right\|_{L^{1}(\Omega)} \leq\left\|\frac{f^{\prime}\left(y_{\varepsilon}^{0}\right) w}{f\left(y_{\varepsilon}^{0}\right)}\right\|_{L^{\infty}(\Omega)}\left\|f^{\prime}\left(y_{\varepsilon}^{0}\right) w\right\|_{L^{1}(\Omega)} \stackrel{\text { by }(58)}{<}+\infty, \\
& \left\|\frac{r^{2}(w, \lambda)}{f\left(y_{\varepsilon}^{0}\right)}\right\|_{L^{p}(\Omega)} \leq\left\|\frac{r(w, \lambda)}{f\left(y_{\varepsilon}^{0}\right)}\right\|_{L^{\infty}(\Omega)}\|r(w, \lambda)\|_{L^{p}(\Omega)} \stackrel{\text { by }(59)}{<}+\infty .
\end{aligned}
$$

In order to deduce the asymptotic property $\|r(w, \lambda)\|_{L^{p}(\Omega) \cap H^{-1}(\Omega)}=o(|\lambda|)$ as $\lambda \longrightarrow 0$, we utilize property of $f$. Then, for a given $w \in C_{0}^{\infty}(\Omega)$ and $\lambda>0$ sufficiently small, we have

$$
\left|f\left(y_{\varepsilon}^{0}+\lambda w\right)-f\left(y_{\varepsilon}^{0}\right)\right| \leq C_{M} f\left(y_{\varepsilon}^{0}\right)\left|\frac{f(\lambda w)}{f(0)}-1\right| .
$$

As a result, we see that

$$
f\left(y_{\varepsilon}^{0}+\lambda w\right) \longrightarrow f\left(y_{\varepsilon}^{0}\right) \operatorname{in} \mathrm{L}^{\mathrm{p}}(\Omega) \text { as } \lambda \longrightarrow 0 .
$$

From this and definition of the directional derivative, we finally deduce

$\lim _{\lambda \rightarrow 0}\left\|\frac{r(w, \lambda)}{\lambda}\right\|\left\|_{L^{p}(\Omega)} \stackrel{\operatorname{by}(60)}{=} \lim _{\lambda \rightarrow 0}\right\| \frac{f\left(y_{\varepsilon}^{0}+\lambda w\right)-f\left(y_{\varepsilon}^{0}\right)}{\lambda}-f^{\prime}\left(y_{\varepsilon}^{0}\right) w \|_{L^{p}(\Omega)}$ by the Lebesgue Dominated Theorem

$$
=\left\|\lim _{\lambda \rightarrow 0}\left(\frac{f\left(y_{\varepsilon}^{0}+\lambda w\right)-f\left(y_{\varepsilon}^{0}\right)}{\lambda}-f^{\prime}\left(y_{\varepsilon}^{0}\right) w\right)\right\|_{L^{p}(\Omega)}=0 .
$$

Hence, $\|r(w, \lambda)\|_{L^{p}(\Omega)}=o(|\lambda|)$ as $\lambda \longrightarrow 0$.

In the functional $J_{\varepsilon}$, we will distinguish three terms

$$
J_{\varepsilon}(u, y, z)=J_{1}(u)+J_{2, \varepsilon}(y)+J_{3, \varepsilon}(z),
$$

where

$$
J_{1}(u)=\frac{\alpha}{p} \int_{\Omega}|u|^{p} d x
$$

$J_{2, \varepsilon}(y)=\frac{1}{2} \int_{\Omega}\left|y-y_{d}\right|^{2} d x+\frac{\varepsilon}{2}\left[\left\|\nabla(-\Delta)^{-1} f(y)\right\|_{L^{2}\left(\Omega ; \mathbb{R}^{N}\right)}^{2}+\|f(y)\|_{L^{1}(\Omega)}\right]$, 


$$
J_{3, \varepsilon}(z)=\frac{1}{2 \varepsilon} \int_{\Omega}|\nabla z|^{2} d x
$$

Now, using Lebesgue's convergence theorem and the fact that $\left(u_{\varepsilon}^{0}, y_{\varepsilon}^{0}, z_{\varepsilon}^{0}\right)$ is an optimal triplet, we get

$$
\begin{aligned}
0 \leq & \lim _{\lambda \rightarrow 0} \frac{J_{\varepsilon}\left(u_{\lambda}, y_{\lambda}, z_{\lambda}\right)-J_{\varepsilon}\left(u_{\varepsilon}^{0}, y_{\varepsilon}^{0}, z_{\varepsilon}^{0}\right)}{\lambda} \\
= & \lim _{\lambda \rightarrow 0} \frac{J_{1}\left(u_{\lambda}\right)-J_{1}\left(u_{\varepsilon}^{0}\right)}{\lambda}+\lim _{\lambda \rightarrow 0} \frac{J_{2, \varepsilon}\left(y_{\lambda}\right)-J_{2, \varepsilon}\left(y_{\varepsilon}^{0}\right)}{\lambda} \\
& +\lim _{\lambda \rightarrow 0} \frac{J_{3, \varepsilon}\left(z_{\lambda}\right)-J_{3, \varepsilon}\left(z_{\varepsilon}^{0}\right)}{\lambda}=A_{1}+A_{2}+A_{3},
\end{aligned}
$$

where

$$
\begin{aligned}
A_{1} & =\frac{\alpha}{p} \lim _{\lambda \rightarrow 0} \int_{\Omega} \frac{\left|u_{\lambda}\right|^{p}-\left|u_{\varepsilon}^{0}\right|^{p}}{\lambda} \\
& =\alpha \int_{\Omega}\left|u_{\varepsilon}^{0}\right|^{p-2} u_{\varepsilon}^{0}\left(-\Delta w-\Delta g-f^{\prime}\left(y_{\varepsilon}^{0}\right) w\right) d x, \\
A_{3} & =\frac{1}{2 \varepsilon} \lim _{\lambda \rightarrow 0} \int_{\Omega} \frac{\left|\nabla z_{\lambda}\right|^{2}-\left|\nabla z_{\varepsilon}^{0}\right|^{2}}{\lambda} d x \\
& =\frac{1}{\varepsilon} \int_{\Omega}\left(\nabla z_{\varepsilon}^{0}, \nabla g\right) d x .
\end{aligned}
$$

As for the term $A_{2}$, we notice that

$$
\begin{aligned}
& \lim _{\lambda \rightarrow 0} \int_{\Omega} \frac{\left|y_{\varepsilon}^{0}+\lambda w-y_{d}\right|^{2}-\left|y_{\varepsilon}^{0}-y_{d}\right|^{2}}{2 \lambda} d x \\
& \quad=\int_{\Omega}\left(y_{\varepsilon}^{0}-y_{d}\right) w d x, \\
& \lim _{\lambda \rightarrow 0} \varepsilon \frac{\left\|f\left(y_{\varepsilon}^{0}+\lambda w\right)\right\|_{L^{1}(\Omega)}-\left\|f\left(y_{\varepsilon}^{0}\right)\right\|_{L^{1}(\Omega)}}{2 \lambda} \\
& \quad=\frac{\varepsilon}{2} \lim _{\lambda \rightarrow 0} \int_{\Omega} \frac{f^{2}\left(y_{\varepsilon}^{0}+\lambda w\right)-f^{2}\left(y_{\varepsilon}^{0}\right)}{\lambda\left(\left|f\left(y_{\varepsilon}^{0}+\lambda w\right)\right|+\left|f\left(y_{\varepsilon}^{0}\right)\right|\right)} d x \\
& \stackrel{\operatorname{by}(60)}{=} \frac{\varepsilon}{2} \int_{\Omega} f^{\prime}\left(y_{\varepsilon}^{0}\right) w d x+\frac{\varepsilon}{2} \lim _{\lambda \rightarrow 0} R(\lambda),
\end{aligned}
$$

where

$R(\lambda)=\int_{\Omega} \frac{2 f\left(y_{\varepsilon}^{0}\right) r(w, \lambda)+2 \lambda f^{\prime}\left(y_{\varepsilon}^{0}\right) w r(w, \lambda)+\lambda^{2}\left(f^{\prime}\left(y_{\varepsilon}^{0}\right) w\right)^{2}+r^{2}(w, \lambda)}{\lambda\left(\left|f\left(y_{\varepsilon}^{0}+\lambda w\right)\right|+\left|f\left(y_{\varepsilon}^{0}\right)\right|\right)} d x$.

Here, we have utilized the following obvious equality

$$
\begin{aligned}
& \lim _{\lambda \rightarrow 0} \int_{\Omega} \frac{2 \lambda f\left(y_{\varepsilon}^{0}\right) f^{\prime}\left(y_{\varepsilon}^{0}\right) w}{\lambda\left(\left|f\left(y_{\varepsilon}^{0}+\lambda w\right)\right|+\left|f\left(y_{\varepsilon}^{0}\right)\right|\right)} d x \\
& \quad=\int_{\Omega} \frac{f\left(y_{\varepsilon}^{0}\right)}{\left|f\left(y_{\varepsilon}^{0}\right)\right|} f^{\prime}\left(y_{\varepsilon}^{0}\right) w d x=\int_{\Omega} f^{\prime}\left(y_{\varepsilon}^{0}\right) w d x,
\end{aligned}
$$

and the fact that $\left(f\left(y_{\varepsilon}^{0}\right)\right) /\left|f\left(y_{\varepsilon}^{0}\right)\right|=1$ for the nonnegative function $f$.

Since

$$
2 \lambda f^{\prime}\left(y_{\varepsilon}^{0}\right) w r(w, \lambda) \leq \lambda^{2}\left(f^{\prime}\left(y_{\varepsilon}^{0}\right) w\right)^{2}+r^{2}(w, \lambda),
$$

it follows from (89) that

$$
\begin{aligned}
|R(\lambda)| \leq & \frac{1}{\lambda} \int_{\Omega} \frac{2\left|f\left(y_{\varepsilon}^{0}\right)\right||r(w, \lambda)|+2 \lambda^{2}\left(f^{\prime}\left(y_{\varepsilon}^{0}\right) w\right)^{2}+2 r^{2}(w, \lambda)}{\left|f\left(y_{\varepsilon}^{0}+\lambda w\right)\right|+\left|f\left(y_{\varepsilon}^{0}\right)\right|} d x \\
\leq & \frac{1}{\lambda} \int_{\Omega} \frac{2\left|f\left(y_{\varepsilon}^{0}\right)\right||r(w, \lambda)|+2 \lambda^{2}\left(f^{\prime}\left(y_{\varepsilon}^{0}\right) w\right)^{2}+2 r^{2}(w, \lambda)}{\left|f\left(y_{\varepsilon}^{0}\right)\right|} d x \\
\leq & \frac{2\|r(w, \lambda)\|_{L^{1}(\Omega)}}{\lambda}+2 \lambda\left\|\frac{\left(f^{\prime}\left(y_{\varepsilon}^{0}\right) w\right)^{2} \|}{\left|f\left(y_{\varepsilon}^{0}\right)\right|}\right\|_{L^{1}(\Omega)} \\
& +\frac{1}{\lambda}\left\|\frac{r^{2}(w, \lambda)}{\left|f\left(y_{\varepsilon}^{0}\right)\right|}\right\|_{L^{1}(\Omega)} \stackrel{\text { by }(61)}{=} \frac{1}{\lambda} o(\lambda) .
\end{aligned}
$$

Then, (102) implies that

$$
\lim _{\lambda \rightarrow 0} \frac{\left\|f\left(y_{\varepsilon}^{0}+\lambda w\right)\right\|_{L^{1}(\Omega)}-\left\|f\left(y_{\varepsilon}^{0}\right)\right\|_{L^{1}(\Omega)}}{2 \lambda}=\frac{\varepsilon}{2} \int_{\Omega} f^{\prime}\left(y_{\varepsilon}^{0}\right) w d x .
$$

Since for any element $\zeta \in H^{-1}(\Omega)$, we have $(-\Delta)^{-1} \zeta \epsilon$ $H_{0}^{1}(\Omega)$, it follows that

$$
\begin{aligned}
\| \nabla & (-\Delta)^{-1}(\eta+\zeta)\left\|_{L^{2}\left(\Omega ; \mathbb{R}^{N}\right)}^{2}-\right\| \nabla(-\Delta)^{-1} \eta \|_{L^{2}\left(\Omega ; \mathbb{R}^{N}\right)}^{2} \\
& =2\left(\nabla(-\Delta)^{-1} \eta, \nabla(-\Delta)^{-1} \zeta\right)_{L^{2}\left(\Omega ; \mathbb{R}^{N}\right)}+\left\|\nabla(-\Delta)^{-1} \zeta\right\|_{L^{2}\left(\Omega ; \mathbb{R}^{N}\right)}^{2} \\
& =-2\left\langle\operatorname{div}\left[\nabla(-\Delta)^{-1} \eta\right],(-\Delta)^{-1} \zeta\right\rangle_{H^{-1}(\Omega) ; H_{0}^{1}(\Omega)}+\|\zeta\|_{H^{-1}(\Omega)}^{2} \\
& =2\left\langle\eta,(-\Delta)^{-1} \zeta\right\rangle_{H^{-1}(\Omega) ; H_{0}^{1}(\Omega)}+\|\zeta\|_{H^{-1}(\Omega)}^{2} \\
& =2\left\langle\zeta,(-\Delta)^{-1} \eta\right\rangle_{H^{-1}(\Omega) ; H_{0}^{1}(\Omega)}+\|\zeta\|_{H^{-1}(\Omega)}, \forall \eta \in H^{-1}(\Omega) .
\end{aligned}
$$

Hence,

$$
\begin{aligned}
\lim _{\lambda \rightarrow 0} & \frac{\varepsilon}{2 \lambda}\left[\left\|\nabla(-\Delta)^{-1} f\left(y_{\varepsilon}^{0}+\lambda w\right)\right\|_{L^{2}\left(\Omega ; \mathbb{R}^{N}\right)}^{2}\right. \\
- & \left.\left\|\nabla(-\Delta)^{-1} f\left(y_{\varepsilon}^{0}\right)\right\|_{L^{2}\left(\Omega ; \mathbb{R}^{N}\right)}^{2}\right] \\
& =\varepsilon\left\langle f^{\prime}\left(y_{\varepsilon}^{0}\right) w,(-\Delta)^{-1} f\left(y_{\varepsilon}^{0}\right)\right\rangle_{H^{-1}(\Omega) ; H_{0}^{1}(\Omega)} \\
& =\varepsilon \int_{\Omega}\left[(-\Delta)^{-1} f\left(y_{\varepsilon}^{0}\right)\right] f^{\prime}\left(y_{\varepsilon}^{0}\right) w d x .
\end{aligned}
$$

As a result, utilizing relations (101), (107), and (109), we obtain 


$$
A_{2}=\int_{\Omega}\left[y_{\varepsilon}^{0}-y_{d}+\frac{\varepsilon}{2} f^{\prime}\left(y_{\varepsilon}^{0}\right)+\varepsilon\left[(-\Delta)^{-1} f\left(y_{\varepsilon}^{0}\right)\right] f^{\prime}\left(y_{\varepsilon}^{0}\right)\right] w d x
$$

From the linearity of $A_{1}, A_{2}$, and $A_{3}$ with respect to $w$ and $g$, we deduce from (98), (99), (100), and (110) that

$$
\begin{aligned}
& \int_{\Omega}\left[y_{\varepsilon}^{0}-y_{d}+\frac{\varepsilon}{2} f^{\prime}\left(y_{\varepsilon}^{0}\right)+\varepsilon\left[(-\Delta)^{-1} f\left(y_{\varepsilon}^{0}\right)\right] f^{\prime}\left(y_{\varepsilon}^{0}\right)\right] w d x \\
& +\alpha \int_{\Omega}\left|u_{\varepsilon}^{0}\right|^{p-2} u_{\varepsilon}^{0}\left(-\Delta w-f^{\prime}\left(y_{\varepsilon}^{0}\right) w\right) d x \\
& +\frac{1}{\varepsilon} \int_{\Omega}\left(\nabla z_{\varepsilon}^{0}, \nabla g\right) d x-\alpha \int_{\Omega}\left|u_{\varepsilon}^{0}\right|^{p-2} u_{\varepsilon}^{0} \Delta g d x=0,
\end{aligned}
$$

for every $w \in C_{0}^{\infty}(\Omega)$ and $g \in C_{0}^{\infty}(\Omega)$.

Let us set $\alpha\left|u_{\varepsilon}^{0}\right|^{p-2} u_{\varepsilon}^{0}=\mu_{\varepsilon}$. Then, (111) implies that

$$
\begin{aligned}
& \int_{\Omega}\left[y_{\varepsilon}^{0}-y_{d}+\frac{\varepsilon}{2} f^{\prime}\left(y_{\varepsilon}^{0}\right)+\varepsilon\left[(-\Delta)^{-1} f\left(y_{\varepsilon}^{0}\right)\right] f^{\prime}\left(y_{\varepsilon}^{0}\right)\right. \\
& \left.-\mu_{\varepsilon} f^{\prime}\left(y_{\varepsilon}^{0}\right)\right] w d x+\int_{\Omega} \mu_{\varepsilon}(-\Delta w) d x=0, \forall w \in C_{0}^{\infty}(\Omega),
\end{aligned}
$$

$$
\frac{1}{\varepsilon} \int_{\Omega}\left(\nabla z_{\varepsilon}^{0}, \nabla g\right) d x-\int_{\Omega} \mu_{\varepsilon} \Delta g d x=0, \forall g \in C_{0}^{\infty}(\Omega) .
$$

From the last equality, we immediately deduce that $\mu_{\varepsilon}=-(1 / \varepsilon) z_{\varepsilon}^{0}+d$, where $d_{\varepsilon} \in C^{2}(\Omega)$ is a weakly harmonic function (it satisfies Laplace's equation $\Delta d=0$ in the sense of distributions). As a consequence, we have $\mu_{\varepsilon} \in H^{1}(\Omega)$.

Taking into account that $C_{0}^{\infty}(\Omega)$ is dense in $H_{0}^{1}(\Omega)$, we see that relations (112)-(113) can be rewritten as follows.

$$
\begin{gathered}
\int_{\Omega}\left[y_{\varepsilon}^{0}-y_{d}+\frac{\varepsilon}{2} f^{\prime}\left(y_{\varepsilon}^{0}\right)+\varepsilon\left[(-\Delta)^{-1} f\left(y_{\varepsilon}^{0}\right)\right] f^{\prime}\left(y_{\varepsilon}^{0}\right)\right] w d x \\
-\int_{\Omega} \mu_{\varepsilon} f^{\prime}\left(y_{\varepsilon}^{0}\right) w d x+<-\Delta \mu_{\varepsilon}, w>_{H^{-1}(\Omega) ; H_{0}^{1}(\Omega)} d x \\
=0, \mu_{\varepsilon}=-\frac{1}{\varepsilon} z_{\varepsilon}^{0}+d \text { a.e.in } \Omega,
\end{gathered}
$$

for every $w \in C_{0}^{\infty}(\Omega)$ and $g \in C_{0}^{\infty}(\Omega)$.

Thus, we can summarize the obtained result as follows.

Theorem 11. Let $\Omega$ be a bounded open domain in $\mathbb{R}^{N}$ with $N \geq 1$. For a given $p \geq 2$, let $\mathscr{K}$ be the subset defined as in (81). Assume that $f: \mathbb{R} \longrightarrow[0,+\infty)$ is a monotonically increasing function such that $f \in C^{1}(\mathbb{R})$ and this function is convex and satisfies property (80). If $\left(u_{\varepsilon}^{0}, y_{\varepsilon}^{0}, z_{\varepsilon}^{0}\right) \in U_{\partial} \times \mathscr{K} \times$ $H_{0}^{1}(\Omega)$ is an optimal solution to the problem (79), (33)-(35), then

$$
\begin{gathered}
-\Delta z_{\varepsilon}^{0}=\Delta y_{\varepsilon}^{0}+f\left(y_{\varepsilon}^{0}\right)+u_{\varepsilon}^{0} \text { in } \Omega \\
z_{\varepsilon}^{0}=0 \text { on } \partial \Omega
\end{gathered}
$$

and setting $\mu_{\varepsilon}=\alpha\left|u_{\varepsilon}^{0}\right|^{p-2} u_{\varepsilon}^{0}$, one has $\mu_{\varepsilon}=-(1 / \varepsilon) z_{\varepsilon}^{0}+d$ a.e. in $\Omega$, and

$$
\begin{gathered}
y_{\varepsilon}^{0}-y_{d}+\frac{\varepsilon}{2} f^{\prime}\left(y_{\varepsilon}^{0}\right)+\varepsilon\left[(-\Delta)^{-1} f\left(y_{\varepsilon}^{0}\right)\right] f^{\prime}\left(y_{\varepsilon}^{0}\right) \\
-\mu_{\varepsilon} f^{\prime}\left(y_{\varepsilon}^{0}\right)=\Delta \mu_{\varepsilon} \quad \text { in } \mathscr{D}^{\prime}(\Omega), \\
\Delta d=0 \quad \text { in } \mathscr{D}^{\prime}(\Omega) .
\end{gathered}
$$

\section{Asymptotic Analysis of Regularized Optimal Control Problem}

Our main aim in this section is find out whether the original optimal control problem (1)-(4) is solvable under assumptions (a)-(c) and its optimal solutions can be attained (in some sense) by optimal solutions to the regularized problem (32)-(35).

The key point of our consideration is that, in contrast to the well-known approaches (see, for instance, $[20,27,28]$ ), we do not assume here the fulfillment of the "standard" extra properties such that the domain $\Omega$ is an open subset of $\mathbb{R}^{N}$ with $N>2$, this domain should be star-shaped and exists a weak solution $y \in H_{0}^{1}(\Omega)$ of Dirichlet problem (2)-(3) satisfy$\operatorname{ing} f(y) \in L^{2}(\Omega)$. Because of this, the existence of at least one optimal pair to the problem (1)-(4) is an open question provided we restrict our consideration only by assumptions (a) $-(\mathrm{c})$.

In what follows, in order to guarantee the consistency of the original problem (1)-(4), we accept the following hypothesis.

Hypothesis 12. The set of feasible solutions $\Xi$ to the problem (1)-(4) is nonempty.

It is worth to notice that the verification of Hypothesis 12 is not too restrictive from practical implementation point of view. Indeed, let $\tilde{y} \in C_{0}^{\infty}(\Omega)$ be an arbitrary function. Then, it is clear that $\tilde{y} \in H_{0}^{1}(\Omega)$ and $f(\tilde{y}) \in L^{1}(\Omega)$, that is, $y \in H_{f}$. Let us define the control $\tilde{u} \in L^{p}(\Omega)$ as follows $\tilde{u}=-\Delta \tilde{y}-f(\tilde{y})$ in $\Omega$. Then, $(\tilde{u}, \tilde{y})$ is a feasible pair to the problem (1)-(4) if only $\tilde{u} \in U_{\partial}$. So, this hypothesis is obviously true if we do not impose any additional restrictions on the class of admissible controls, i.e., $U_{\partial}=L^{p}(\Omega)$.

The following result is crucial in this paper and it shows that solvability of the original OCP (1)-(4) in some sense is equivalent to its consistency, i.e., OCP (1)-(4) admits at least one solution if and only if Hypothesis 12 is fulfilled. However, in order to establish this fact, we apply an indirect approach based on the variant of Tikhonov regularization which is described in Section 4.

Theorem 13. Let $\Omega$ be a bounded open domain in $\mathbb{R}^{N}$ with $N \geq 1$, let $U_{\partial}$ be a nonempty closed convex subset of $L^{p}(\Omega)$, $2 \leq p<+\infty$, and let $f: \mathbb{R} \longrightarrow[0,+\infty)$ be a monotonically 
increasing function such that $f \in C(\mathbb{R})$. Let $\left\{\left(u_{\varepsilon}^{0}, y_{\varepsilon}^{0}, z_{\varepsilon}^{0}\right) \in \Lambda_{\varepsilon}\right\}_{\varepsilon \rightarrow 0}$ be a sequence of optimal solutions to regularized problems (32)-(35) when the parameter $\varepsilon>0$ varies in a strictly decreasing sequence of positive numbers converging to 0 . Assume that Hypothesis 12 holds true and the sequence $\left\{f\left(y_{\varepsilon}^{0}\right)\right\}_{\varepsilon \rightarrow 0}$ is bounded in $L^{1}(\Omega) \cap H^{-1}(\Omega)$. Then, there is a subsequence of $\left\{\left(u_{\varepsilon}^{0}, y_{\varepsilon}^{0}, z_{\varepsilon}^{0}\right)\right\}_{\varepsilon \rightarrow 0}$, still denoted by the suffix $\varepsilon$, such that

$$
\begin{aligned}
u_{\varepsilon}^{0} & \rightarrow u^{0} i n L^{p}(\Omega), y_{\varepsilon}^{0} \rightarrow y^{0} i n H_{0}^{1}(\Omega), z_{\varepsilon}^{0} \longrightarrow 0 \operatorname{inH} H_{0}^{1}(\Omega),-\Delta y^{0} \\
& =f\left(y^{0}\right)+u^{0} \text { in } \mathscr{D}(\Omega), u \in U_{\partial},\left(u^{0}, y^{0}\right) \\
& \in \Xi, J\left(u^{0}, y^{0}\right)=\inf _{(u, y) \in \Xi} J(u, y) .
\end{aligned}
$$

Proof. Since $\Xi \neq \varnothing$, it follows that, for a given $\delta>0$, there exists $\left(u_{\delta}^{*}, y_{\delta}^{*}\right)$ such that $\left(u_{\delta}^{*}, y_{\delta}^{*}\right)$ is a feasible solution to optimal control problem (1)-(4), and

$$
\begin{aligned}
\inf _{(u, y) \in \Xi} J(u, y) & =\inf _{(u, y) \in \Xi}\left[\frac{1}{2} \int_{\Omega}\left|y-y_{d}\right|^{2} d x+\frac{\alpha}{p} \int_{\Omega}|u|^{p} d x\right] \\
& \geq J\left(u_{\delta}^{*}, y_{\delta}^{*}\right)-\delta .
\end{aligned}
$$

Hence, in view of Definition 5 and Lemma 2, $u_{\delta}^{*} \in U_{\partial}$, $y_{\delta}^{*} \in H_{0}^{1}(\Omega), f\left(y_{\delta}^{*}\right) \in L^{1}(\Omega) \cap H^{-1}(\Omega)$, and the pair $\left(u_{\delta}^{*}, y_{\delta}^{*}\right)$ is related by integral identity (8). Therefore, for each $\varepsilon>0$, the triplet $\left(u_{\delta}^{*}, y_{\delta}^{*}, 0\right)$ is a feasible solution for regularized problem (32)-(35), i,e, $\left(u_{\delta}^{*}, y_{\delta}^{*}, 0\right) \in \Lambda_{\varepsilon}$ for all $\varepsilon>0$. Taking this fact into account, we see that

$$
\begin{aligned}
J_{\varepsilon}\left(u_{\varepsilon}^{0}, y_{\varepsilon}^{0}, z_{\varepsilon}^{0}\right)= & \frac{1}{2} \int_{\Omega}\left|y_{\varepsilon}^{0}-y_{d}\right|^{2} d x+\frac{\alpha}{p} \int_{\Omega}\left|u_{\varepsilon}^{0}\right|^{p} d x \\
& +\frac{1}{2 \varepsilon} \int_{\Omega}\left|\nabla z_{\varepsilon}^{0}\right|^{2} d x+\frac{\varepsilon}{2} \\
& \cdot\left[\left\|f\left(y_{\varepsilon}^{0}\right)\right\|_{H^{-1}(\Omega)}^{2}+\left\|f\left(y_{\varepsilon}^{0}\right)\right\|_{L^{1}(\Omega)}\right] \\
= & \inf _{(u, y, z) \in \Lambda_{\varepsilon}} J_{\varepsilon}(u, y, z) \leq J_{\varepsilon}\left(u_{\delta}^{*}, y_{\delta}^{*}, 0\right) \\
= & \frac{1}{2} \int_{\Omega}\left|y_{\delta}^{*}-y_{d}\right|^{2} d x+\frac{\alpha}{p} \int_{\Omega}\left|u_{\delta}^{*}\right|^{p} d x \\
& +\frac{\varepsilon}{2}\left[\left\|f\left(y_{\delta}^{*}\right)\right\|_{H^{-1}(\Omega)}^{2}+\left\|f\left(y_{\delta}^{*}\right)\right\|_{L^{1}(\Omega)}\right] \\
= & C_{1}+\varepsilon C_{2}<+\infty,
\end{aligned}
$$

where

$$
\begin{aligned}
& C_{1}:=\frac{1}{2} \int_{\Omega}\left|y_{\delta}^{*}-y_{d}\right|^{2} d x+\frac{\alpha}{p} \int_{\Omega}\left|u_{\delta}^{*}\right|^{p} d x, \\
& C_{2}:=\frac{1}{2}\left[\left\|f\left(y_{\delta}^{*}\right)\right\|_{H^{-1}(\Omega)}^{2}+\left\|f\left(y_{\delta}^{*}\right)\right\|_{L^{1}(\Omega)}\right],
\end{aligned}
$$

and (see (118))

$$
\begin{aligned}
& \frac{1}{2} \int_{\Omega}\left|y_{\varepsilon}^{0}-y_{d}\right|^{2} d x+\frac{\alpha}{p} \int_{\Omega}\left|u_{\varepsilon}^{0}\right|^{p} d x \\
& \quad \geq \frac{1}{2} \int_{\Omega}\left|y_{\delta}^{*}-y_{d}\right|^{2} d x+\frac{\alpha}{p} \int_{\Omega}\left|u_{\delta}^{*}\right|^{p} d x-\delta .
\end{aligned}
$$

Since this relation holds true for each $\varepsilon>0$ varying in a given interval $\left(0, \varepsilon^{0}\right]$ and each $\delta>0$, it follows that

$$
\begin{aligned}
& \sup _{\varepsilon \in\left(0, \varepsilon^{0}\right]}\left\|u_{\varepsilon}^{0}\right\|_{L^{p}(\Omega)}^{p} \leq \frac{\left(C_{1}+\delta\right) p}{\alpha}, \sup _{\varepsilon \in\left(0, \varepsilon^{0}\right]}\left[\frac{1}{\varepsilon}\left\|z_{\varepsilon}^{0}\right\|_{H_{0}^{1}(\Omega)}^{2}\right] \\
& \leq 2\left(C_{1}+\varepsilon^{0} C_{2}\right), \\
& \sup _{\varepsilon \in\left(0, \varepsilon^{0}\right]}\left\|y_{\varepsilon}^{0}\right\|_{L^{2}(\Omega)}^{2} \leq 4\left(C_{1}+\delta\right)+2\left\|y_{d}\right\|_{L^{2}(\Omega)}^{2} .
\end{aligned}
$$

In addition, the sequence $\left\{f\left(y_{\varepsilon}^{0}\right)\right\}_{\varepsilon \in\left(0, \varepsilon^{0}\right]}$ is assumed to be bounded in $L^{1}(\Omega) \cap H^{-1}(\Omega)$. Taking this fact into account, we deduce that

$$
\sup _{\varepsilon \in\left(0, \varepsilon^{0}\right]}\left\|f\left(y_{\varepsilon}^{0}\right)\right\|_{H^{-1}(\Omega)}^{2} \leq 2 C_{2}+C_{1}, \sup _{\varepsilon \in\left(0, \varepsilon^{0}\right]}\left\|f\left(y_{\varepsilon}^{0}\right)\right\|_{L^{1}(\Omega)} \leq 2 C_{2}+C_{1} .
$$

Hence, the sequences $\left\{u_{\varepsilon}^{0}\right\}_{\varepsilon \in\left(0, \varepsilon^{0}\right]}, \quad\left\{y_{\varepsilon}^{0}\right\}_{\varepsilon \in\left(0, \varepsilon^{0}\right]}$, and $\left\{f\left(y_{\varepsilon}^{0}\right)\right\}_{\varepsilon \in\left(0, \varepsilon^{0}\right]}$ are weakly compact in $L^{p}(\Omega), L^{2}(\Omega)$, and $H^{-1}(\Omega)$, respectively, whereas estimate (122) implies that the sequence $\left\{z_{\varepsilon}^{0}\right\}_{\varepsilon \rightarrow 0}$ is strongly convergent to 0 in $H_{0}^{1}(\Omega)$. So, we can suppose that there exist elements $u^{0} \in L^{p}(\Omega), y$ $\in L^{2}(\Omega), \xi \in H^{-1}(\Omega)$, and a sequence $\left\{\varepsilon_{k}\right\}_{k \in \mathbb{R}}$ monotonically converging to zero as $k \longrightarrow \infty$ such that

$$
\begin{gathered}
u_{\varepsilon_{k}}^{0} \longrightarrow u^{0} \text { weakly in } L^{\mathrm{p}}(\Omega), \\
y_{\varepsilon_{k}}^{0} \longrightarrow y^{0} \text { weakly in } L^{2}(\Omega), \\
f\left(y_{\varepsilon_{k}}^{0}\right) \longrightarrow \xi \text { weakly in } H^{-1}(\Omega), \\
z_{\varepsilon_{k}} \longrightarrow 0 \text { strongly in } H_{0}^{1}(\Omega) \text { ask } \longrightarrow \infty .
\end{gathered}
$$

Let us show that, in fact, we have the weak convergence $y_{\varepsilon_{k}}^{0} \rightarrow y^{0}$ in $H_{0}^{1}(\Omega)$. Indeed, arguing as in the proof of Theorem 8 , we utilize the integral identity

$$
\begin{gathered}
\int_{\Omega}\left[\left|\nabla y_{\varepsilon_{k}}^{0}\right|^{2}+\left(\nabla z_{\varepsilon_{k}}^{0}, \nabla y_{\varepsilon_{k}}^{0}\right)-u_{\varepsilon_{k}}^{0} y_{\varepsilon_{k}}^{0}\right] d x \\
=\left\langle f\left(y_{\varepsilon_{k}}^{0}\right), y_{\varepsilon_{k}}^{0}\right\rangle_{H^{-1}(\Omega) ; H_{0}^{1}(\Omega)},
\end{gathered}
$$

which holds true for each $\varepsilon \in\left(0, \varepsilon^{0}\right]$ and reflects the fact that the triplets $\left\{\left(u_{\varepsilon_{k}}^{0}, y_{\varepsilon_{k}}^{0}, z_{\varepsilon_{k}}^{0}\right)\right\}_{k \in \mathbb{R}}$ are feasible to the problem 
(32)-(35) for each $k \in \mathbb{R}$. Then, we deduce from (129) that

$$
\begin{aligned}
\left\|\nabla y_{\varepsilon_{k}}^{0}\right\|_{L^{2}(\Omega)^{N}}^{2} \leq & {\left[\left\|z_{\varepsilon_{k}}^{0}\right\|_{H_{0}^{1}(\Omega)}+\left\|f\left(y_{\varepsilon_{k}}^{0}\right)\right\|_{H^{-1}(\Omega)}\right.} \\
& \left.+C_{\Omega}|\Omega|^{(p-2) / 2 p}\left\|u_{\varepsilon_{k}}^{0}\right\|_{L^{p}(\Omega)}\right]\left\|\nabla y_{\varepsilon_{k}}^{0}\right\|_{L^{2}(\Omega)^{N}} .
\end{aligned}
$$

Hence, estimates (122)-(124) imply that

$$
\begin{aligned}
\sup _{k \in \mathbb{N}}\left\|\nabla y_{\varepsilon_{k}}^{0}\right\|_{L^{2}(\Omega)^{N}} \leq & {\left[\sqrt{2 \varepsilon^{0}\left(C_{1}+\varepsilon^{0} C_{2}\right)}+\sqrt{2 C_{2}}\right.} \\
& \left.+C_{\Omega}|\Omega|^{(p-2) / 2 p} \sqrt{\frac{\left(C_{1}+\delta\right) p}{\alpha}}\right]=: C^{*}<+\infty .
\end{aligned}
$$

Thus, without loss of generality, we can suppose that (up to a subsequence)

$y_{\varepsilon_{k}}^{0} \rightarrow y^{0}$ in $H_{0}^{1}(\Omega), y_{\varepsilon_{k}}^{0} \longrightarrow y^{0}$ in $L^{2}(\Omega), y_{\varepsilon_{k}}^{0}(x) \longrightarrow y^{0}(x)$ a.e.in $\Omega$.

Utilizing the pointwise convergence $(132)_{3}$ and (c)-property, we see that $f\left(y_{\varepsilon_{k}}^{0}\right) \longrightarrow f\left(y^{0}\right)$ almost everywhere in $\Omega$ as $k \longrightarrow \infty$. Let us show that, in fact, we have the strong convergence

$$
f\left(y_{\varepsilon_{k}}^{0}\right) \longrightarrow f\left(y^{0}\right) \operatorname{in} L^{1}(\Omega) \text { as } k \longrightarrow \infty
$$

and, as a consequence of (127), $\xi=f\left(y^{0}\right)$ and $f\left(y^{0}\right) \in H^{-1}($ $\Omega) \cap L^{1}(\Omega)$.

To this end, we make use of some arguments of the proof of Theorem 8. With that in mind, for each $k \in \mathbb{N}$, we make use of the decomposition $y_{\varepsilon_{k}}^{-}=y_{\varepsilon_{k}}^{+}-y_{\varepsilon_{k}}^{-}$with

$$
y_{\varepsilon_{k}}^{+}=\max \left\{0,-y_{\varepsilon_{k}}\right\} \in H_{0}^{1}(\Omega), y_{\varepsilon_{k}}^{-}=\max \left\{0,-y_{\varepsilon_{k}}\right\} \in H_{0}^{1}(\Omega),
$$

and set $T_{\eta}(y)(x)=\left\{\begin{array}{ll}\eta, & \text { if } y(x)>\eta, \\ y(x), & \text { if } y(x) \leq \eta .\end{array}\right.$ Then, $T_{\eta}(y) \in L^{\infty}($ $\Omega) \cap H_{0}^{1}(\Omega)$ for all $y \in H_{0}^{1}(\Omega)$ such that $y \geq 0$. Using $y_{\varepsilon, k}^{+} \in$ $H_{0}^{1}(\Omega)$ as a test function in (25), we find that

$$
\begin{aligned}
\int_{\Omega} f\left(y_{\varepsilon, k}\right) T_{\eta}\left(y_{\varepsilon, k}^{+}\right) d x= & \int_{\Omega}\left(\nabla z_{\varepsilon, k}, \nabla T_{\eta}\left(y_{\varepsilon, k}^{+}\right)\right) d x \\
& +\int_{\Omega}\left(\nabla y_{\varepsilon, k}, \nabla T_{\eta}\left(y_{\varepsilon, k}^{+}\right)\right) d x \\
& -\int_{\Omega} u_{\varepsilon, k} T_{\eta}\left(y_{\varepsilon, k}^{+}\right) d x, \forall \eta \in \mathbb{N} .
\end{aligned}
$$

Since $T_{\eta}\left(y_{\varepsilon, k}^{+}\right) \longrightarrow y_{\varepsilon, k}^{+}$in $H_{0}^{1}(\Omega)$ as $\eta \longrightarrow \infty$, the limit passage in (135) as $\eta \longrightarrow \infty$ leads to the relation

$$
\begin{aligned}
\int_{\Omega} f\left(y_{\varepsilon_{k}}\right) y_{\varepsilon_{k}}^{+} d x= & \int_{\Omega}\left(\nabla z_{\varepsilon_{k}}, \nabla y_{\varepsilon_{k}}^{+}\right) d x+\int_{\Omega}\left(\nabla y_{\varepsilon_{k}}, \nabla y_{\varepsilon_{k}}^{+}\right) d x \\
& -\int_{\Omega} u_{\varepsilon_{k}} y_{\varepsilon_{k}}^{+} d x .
\end{aligned}
$$

From this and the fact that $\left\|y_{\varepsilon_{k}}^{+}\right\|_{H_{0}^{1}(\Omega)} \leq\left\|y_{\varepsilon_{k}}\right\|_{H_{0}^{1}(\Omega)}$, we deduce the estimate

$$
\begin{aligned}
& \int_{\Omega} f\left(y_{\varepsilon_{k}}\right) y_{\varepsilon_{k}}^{+} d x \leq {\left[\left\|z_{\varepsilon_{k}}\right\|_{H_{0}^{1}(\Omega)}+\left\|y_{\varepsilon_{k}}\right\|_{H_{0}^{1}(\Omega)}\right.} \\
&\left.+C_{\Omega}|\Omega|^{(p-2) / 2 p}\left\|u_{\varepsilon_{k}}\right\|_{L^{p}(\Omega)}\right]\left\|y_{\varepsilon_{k}}\right\|_{H_{0}^{1}(\Omega)} \\
& \stackrel{\operatorname{by}(84),(84)(76)}{\leq} C^{*}\left[\sqrt{2\left(C_{1}+\varepsilon^{0} C_{2}\right)}+C^{*}\right. \\
&\left.+C_{\Omega}|\Omega|^{(p-2) / 2 p}\left(\frac{\left(C_{1}+\delta\right) p}{\alpha}\right)^{1 / p}\right] .
\end{aligned}
$$

Arguing in a similar manner, it can be shown that

$$
\begin{aligned}
\int_{\Omega} f\left(y_{\varepsilon_{k}}\right) y_{\varepsilon_{k}}^{-} d x \leq & C^{*}\left[\sqrt{2\left(C_{1}+\varepsilon^{0} C_{2}\right)}+C^{*}\right. \\
& \left.+C_{\Omega}|\Omega|^{(p-2) / 2 p}\left(\frac{\left(C_{1}+\delta\right) p}{\alpha}\right)^{1 / p}\right] .
\end{aligned}
$$

Since $y_{\varepsilon_{k}}^{+}+y_{\varepsilon_{k}}^{-}=\left|y_{\varepsilon_{k}}\right|$, it follows from (137)-(138) that there exists a positive constant $\widehat{C}$, independent of $\varepsilon$ and $y_{\varepsilon_{k}}$ such that

$$
\sup _{k \in \mathbb{N}}\left\|f\left(y_{\varepsilon_{k}}\right) y_{\varepsilon_{k}}\right\|_{L^{1}(\Omega)} \leq \widehat{C}
$$

In order to prove the strong convergence (133), it remains to make use of Vitali's theorem. We fix an arbitrary $\zeta>0$ and take $m>0$ and $\tau>0$ such that $m>2 \widehat{C} / \zeta, \tau=\zeta /(2 f$ $(m))$. Then, for every measurable set $S \subset \Omega$ with Lebesgue measure $|S|<\zeta$, we have

$$
\begin{aligned}
\int_{S} f\left(y_{\varepsilon_{k}}\right) d x & =\int_{\left\{x \in S: y_{\varepsilon_{k}}(x)>m\right\}} f\left(y_{\varepsilon_{k}}\right) d x+\int_{\left\{x \in S: y_{\varepsilon_{k}}(x) \leq m\right\}} f\left(y_{\varepsilon_{k}}\right) d x \\
& \leq \frac{1}{m} \int_{\left\{x \in S: y_{\varepsilon_{k}}(x)>m\right\}} y_{\varepsilon_{k}} f\left(y_{\varepsilon_{k}}\right) d x+\int_{\left\{x \in S: y_{\varepsilon_{k}}(x) \leq m\right\}} f(m) d x \\
& \leq \frac{1}{m} \int_{\Omega}\left|y_{\varepsilon_{k}}\right| f\left(y_{\varepsilon_{k}}\right) d x+f(m)|S| \stackrel{\text { by }(91) \widehat{C}}{\leq} \frac{\widehat{C}}{m}+f(m)|S| \leq \frac{\zeta}{2}+\frac{\zeta}{2} .
\end{aligned}
$$


As a result, we see that the sequence $\left\{f\left(y_{\varepsilon_{k}}\right)\right\}_{k \in \mathbb{N}}$ is equiintegrable and, hence, the desired convergence (133) is a direct consequence of the pointwise convergence $f\left(y_{\varepsilon_{k}}^{0}\right)$ $\longrightarrow f\left(y^{0}\right)$ almost everywhere in $\Omega$ and Vitali's convergence theorem.

We are now in a position to show that $\left(u^{0}, y^{0}\right)$ is a feasible solution to the original OCP (1)-(4). Indeed, in view of the initial assumptions, the set $U_{\partial}$ is sequentially closed with respect to the weak topology of $L^{p}(\Omega)$. Hence, $u^{0} \in U_{\partial}$. It remains to show that the pair $\left(u^{0}, y^{0}\right)$ is related by the integral identity (8). To this end, we note that $\left(u_{\varepsilon_{k}}^{0}, y_{\varepsilon_{k}}^{0}, z_{\varepsilon_{k}}^{0}\right) \in$ $\Lambda_{\varepsilon_{k}}$ for all $k \in \mathbb{R}$. Hence, the equality

$$
\begin{gathered}
\int_{\Omega}\left[\left(\nabla y_{\varepsilon, k}, \nabla \varphi\right)+\left(\nabla z_{\varepsilon, k}, \nabla \varphi\right)-u_{\varepsilon, k} \varphi\right] d x \\
=\left\langle f\left(y_{\varepsilon, k}\right), \varphi\right\rangle_{H^{-1}(\Omega) ; H_{0}^{1}(\Omega)},
\end{gathered}
$$

holds true for each test function $\varphi \in C_{0}^{\infty}(\Omega)$. As a result, the limit passage in (141) as $k \longrightarrow \infty$ becomes trivial, and it immediately leads us to the expected integral identity (8). Thus, combining all properties of the pair $\left(u^{0}, y^{0}\right)$ established here and before, we finally deduce that $\left(u^{0}, y^{0}\right) \in \Xi$.

To conclude the proof, we have to show that $\left(u^{0}, y^{0}\right) \in \Xi$ in an optimal pair to the problem (1)-(4). To do so, we assume the converse, namely, there is a pair $(\widehat{u}, \widehat{y}) \in \Xi$ such that $J(\widehat{u}, \widehat{y})<J\left(u^{0}, y^{0}\right)$. Then, the triplet $(\widehat{u}, \widehat{y}, 0)$ is feasible to the regularized problem (i.e., $(\widehat{u}, \hat{y}, 0)) \in \Lambda_{\varepsilon_{k}}$ for each $k \in$ N. Hence,

$$
\begin{aligned}
& J(\widehat{u}, \widehat{y})+\frac{\varepsilon_{k}}{2}\left[\|f(\widehat{y})\|_{H^{-1}(\Omega)}^{2}+\|f(\widehat{y})\|_{L^{1}(\Omega)}\right] \\
& =J_{\varepsilon_{k}}(\widehat{u}, \widehat{y}, 0) \geq \inf _{(u, y, z) \in \Lambda_{\varepsilon_{k}}} J_{\varepsilon_{k}}(u, y, z) \\
& =J_{\varepsilon_{k}}\left(u_{\varepsilon_{k}}^{0}, y_{\varepsilon_{k}}^{0}, z_{\varepsilon_{k}}^{0}\right), \forall k \in \mathbb{N} .
\end{aligned}
$$

Therefore, passing in (142) to the limit as $k \longrightarrow \infty$ and using the properties

$$
\begin{aligned}
& \liminf _{k \rightarrow \infty} \int_{\Omega}\left|y_{\varepsilon_{k}}^{0}-y_{d}\right|^{2} d x \stackrel{\text { by (85) }}{=} \int_{\Omega}\left|y^{0}-y_{d}\right|^{2} d x, \\
& \liminf _{k \rightarrow \infty} \int_{\Omega}\left|u_{\varepsilon_{k}}^{0}\right|^{p} d x \stackrel{\text { by(85)(79) }}{\geq} \int_{\Omega}\left|u^{0}\right|^{p} d x, \\
& \lim _{k \rightarrow \infty}\left[\frac{1}{\varepsilon_{k}}\left\|z_{\varepsilon_{k}}^{0}\right\|_{H_{0}^{1}(\Omega)}^{2}\right] \stackrel{\text { by }(85)(79)(76)}{=} \text { const }>0 \text {, } \\
& \liminf _{k \rightarrow \infty}\left[\frac{\varepsilon_{k}}{2}\left\|f\left(y_{\varepsilon_{k}}^{0}\right)\right\|_{H^{-1}(\Omega)}^{2}\right] \stackrel{\text { by (85)(79)(76)(81) }}{=} 0 \text {, } \\
& \lim _{k \rightarrow \infty}\left[\frac{\varepsilon_{k}}{2}\left\|f\left(y_{\varepsilon_{k}}^{0}\right)\right\|_{L^{1}(\Omega)}\right] \stackrel{\operatorname{by}(85)(79)(76)(81)(86)}{=} 0,
\end{aligned}
$$

we obtain

$$
\begin{aligned}
J(\widehat{u}, \widehat{y}) & \geq \liminf _{k \rightarrow \infty} J_{\varepsilon_{k}}\left(u_{\varepsilon_{k}}^{0}, y_{\varepsilon_{k}}^{0}, z_{\varepsilon_{k}}^{0}\right) \\
& \geq J\left(u^{0}, y^{0}\right)+\lim _{k \rightarrow \infty}\left[\frac{1}{\varepsilon_{k}}\left\|z_{\varepsilon_{k}}^{0}\right\|_{H_{0}^{1}(\Omega)}^{2}\right] \geq J\left(u^{0}, y^{0}\right) .
\end{aligned}
$$

As a result, it leads us to a contradiction. Thus, $\left(u^{0}, y^{0}\right)$ $\epsilon \Xi$ in an optimal pair to the problem (1)-(4).

To the end of proof, we note if the original OCP admits a unique solution, then the asymptotic analysis given before remains valid for each subsequence $\left\{\left(u_{\varepsilon_{k}}^{0}, y_{\varepsilon_{k}}^{0}, z_{\varepsilon_{k}}^{0}\right)\right\}_{k \in \mathbb{R}}$ of the sequence of optimal solutions $\left\{\left(u_{\varepsilon}^{0}, y_{\varepsilon}^{0}, z_{\varepsilon}^{0}\right) \in \Lambda_{\varepsilon}\right\}_{\varepsilon \rightarrow 0}$. Therefore, the limits in (125)-(128) do not depend on the choice of a subsequence, and, hence, $\left(u^{0}, y^{0}\right) \in \Xi$ is a unique limit pair for the entire sequence of optimal triplets $\left\{\left(u_{\varepsilon}^{0}, y_{\varepsilon}^{0}\right)\right\}_{\varepsilon>0}$.

\section{Data Availability}

All the results in this manuscript are produced by the authors.

\section{Conflicts of Interest}

No potential conflict of interest was reported by the authors.

\section{References}

[1] J.-L. Lions, Optimal Control of Systems Governed by Partial Differential Equations, Springer, Berlin, 1971.

[2] J.-L. Lions, "Some Aspects of the Optimal Control of Distributed Paremeter Systems," in Regional Conference Series in Applied Mathematics, Philadelphia, PA, USA, 1972SIAM.

[3] E. Casas and M. Mateos, Optimal Control of Partial Differential Equations, Computational Mathematics, Numerical Analysis and Applications, Lectures Notes of the XVII J. L. Lions Spanish-French School, M. Mateos and P. Alonso, Eds., SEMA-SIMAI Springer Series, 2017.

[4] A. V. Fursikov, Optimal Control of Distributed Systems. Theory and Applications, Series: Translations of Mathematical Monographs, American Mathematical Soc., 2000.

[5] P. I. Kogut and G. Leugering, Optimal Control Problems for Partial Differential Equations on Reticulated Domains. Approximation and Asymptotic Analysis, Series: Systems and Control, Birkhäuser, Boston, MA, USA, 2011.

[6] I. Lasiecka and R. Triggiani, Control Theory for Partial Differential Equations: Continuous and Approximation Theories. I. Abstract Parabolic Systems, Encyclopedia of Mathematics and its Applications, 74, Cambridge University Press, Cambridge, 2000.

[7] I. Lasiecka and R. Triggiani, Control Theory for Partial Differential Equations: Continuous and Approximation Theories. II. Abstract Hyperbolic-like Systems over a Finite Time Horizon, Encyclopedia of Mathematics and its Applications, 75, Cambridge University Press, Cambridge, 2000.

[8] P. Neittaanmaki, J. Sprekels, and D. Tiba, Optimization of Elliptic Systems. Theory and Applications, Springer Monographs in Mathematics, Springer, New York, NY, USA, 2006. 
[9] F. Tröltzsch, Optimal Control of Partial Differential Equations. Theory, Methods and Applications, Translated from the 2005 German original, J. Sprekels, Ed., American Mathematical Society, Providence, RI, USA, 2010.

[10] I. M. Gelfand, "Some problems in the theory of quasi-linear equations," American Mathematical Society Translations: Series 2, vol. 2, no. 29, pp. 289-292, 1963.

[11] M. G. Crandall and P. H. Rabinowitz, "Some continuation and variational methods for positive solutions of nonlinear elliptic eigenvalue problems," Archive for Rational Mechanics and Analysis, vol. 58, no. 3, pp. 207-218, 1975.

[12] F. Mignot and J. P. Puel, "Sur une classe de problemes non lineaires avec non linearite positive, croissante, convexe," Communications in Partial Differential Equations, vol. 5, no. 8, pp. 791-836, 2007.

[13] T. Gallouët, F. Mignot, and J. P. Puel, "Quelques résultats sur le problème $-\Delta u=\lambda e^{u}$," Comptes rendus de l'Académie des Sciences, vol. 307, pp. 289-292, 1988.

[14] H. Fujita, "On the blowing up of the solutions to the Cauchy problem for $u_{t}=\Delta u+u^{1+\alpha}$," Journal of the Faculty of Science, University of Tokyo. Sect. 1, Mathematics, Astronomy, Physics, Chemistry, vol. 13, pp. 109-124, 1996.

[15] R. G. Pinsky, "Existence and nonexistence of global solutions $u_{t}=\Delta u+a(x) u^{p}$ in $\mathbb{R}^{d}$," Journal of Differential Equations, vol. 133, pp. 152-177, 1997.

[16] R. Ferreira, A. De Pablo, and J. L. Vazquez, "Classification of blow-up with nonlinear diffusion and localized reaction," Journal of Differential Equations, vol. 231, no. 1, pp. 195-211, 2006.

[17] J. Dolbeault and R. Stańczy, "Non-existence and uniqueness results for supercritical semilinear elliptic equations," Annales Henri Poincaré, vol. 10, no. 7, pp. 1311-1333, 2010.

[18] S. Chandrasekhar, An Introduction to the Study of Stellar Structures, Dover Publishing Inc., 1985.

[19] D. A. F. Kamenetskii, Diffusion and Heat Transfer in Chemical Kinetics, Plenum Press, 2nd edition, 1969.

[20] E. Casas, O. Kavian, and J. P. Puel, "Optimal control of an ill-posed elliptic semilinear equation with an exponential nonlinearity," ESAIM: Control, Optimization and Calculus of Variations, vol. 3, pp. 361-380, 1998.

[21] P. I. Kogut, "On Approximation of an optimal boundary control problem for linear elliptic equation with unbounded coefficients," Discrete and Continuous Dynamical Systems, Series A (DCDS-A), vol. 34, no. 5, pp. 2105-2133, 2014.

[22] P. Pedregal, "On variant of Tikhonov regularization in optimal control under PDEs," 2019, https://arxiv.org/abs/1803.09096.

[23] F. Pörnel and D. Wachsmuth, "Tikhonov regularization of optimal control problems governed by semi-linear partial differential equations," Mathematical Control and Related Fields, vol. 8, no. 1, pp. 315-335, 2018.

[24] E. Casas, P. I. Kogut, and G. Leugering, "Approximation of optimal control problems in the coefficient for the \$p\$-Laplace equation. I. Convergence result," SIAM Journal on Control and Optimization, vol. 54, no. 3, pp. 1406-1422, 2016.

[25] P. I. Kogut, "S-convergence of the conditional optimization problems and its variational properties," Problemy Upravleniya I Informatiki (Avtomatika), vol. 4, pp. 64-79, 1997.

[26] P. I. Kogut, "Variational S-convergence of minimization problems. Part I. Definitions and basic properties," Problemy Upravleniya I Informatiki (Avtomatika), vol. 5, pp. 29-42, 1996.
[27] P. I. Kogut and O. P. Kupenko, "On optimal control problem for an ill-posed strongly nonlinear elliptic equation with $\mathrm{p}$ -Laplace operator and $L^{1}$-type of nonlinearity," Discrete and Continuous Dynamical Systems-B, vol. 24, no. 3, pp. 12731295, 2019.

[28] P. I. Kogut and O. P. Kupenko, "On approximation of an optimal control problem for Ill-posed strongly nonlinear elliptic equation with p-Laplace operator," in Modern Mathematics and Mechanics. Fundamentals, Problems and Challenges, V. A. Sadovnichiy and M. Zgurovsky, Eds., vol. 23, pp. 445-480, Springer, 2019.

[29] P. I. Kogut, R. Manzo, and A. O. Putchenko, "On approximate solutions to the Neumann elliptic boundary value problem with non-linearity of exponential type," Boundary Value Problems, vol. 2016, Article ID 208, 2016.

[30] R. Manzo, “On Nuemann boundary control problem for Illposed strongly nonlinear elliptic equation with $p$-Laplace operator and $L^{1}$-type of nonlinearity," Ricerche di Matematica, vol. 68 , no. 2, pp. 769-802, 2019.

[31] R. Manzo, "On Tikhonov regularization of optimal Neumann boundary control problem for an ill-posed strongly nonlinear elliptic equation with an exponential type of non-linearity," Differential and Integral Equations, vol. 33, no. 3-4, pp. 139$162,2020$.

[32] P. I. Kogut and O. P. Kupenko, Approximation Methods in Optimization of Nonlinear Systems, De Gruyter Series in Nonlinear Analysis and Applications, vol. 32, Walter de Gruyter $\mathrm{GmbH}$, Berlin, Boston, 2019.

[33] D. Kinderlehrer and G. Stampacchia, An Introduction to Variational Inequalities and Their Applications, Academic Press, New York, NY, USA, 1980. 